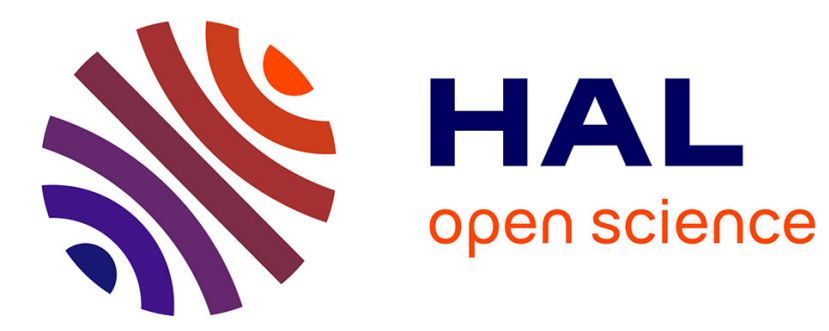

\title{
Un gisement belloisien sur les bords de la Seine: Le Closeau à Rueil-Malmaison (Hauts-de-Seine)
}

Nicolas Teyssandier

\section{To cite this version:}

Nicolas Teyssandier. Un gisement belloisien sur les bords de la Seine: Le Closeau à Rueil-Malmaison (Hauts-de-Seine). Bulletin de la Société préhistorique française, 2000, 97 (2), pp.211-228. halshs00430943

\section{HAL Id: halshs-00430943 \\ https://shs.hal.science/halshs-00430943}

Submitted on 10 Nov 2009

HAL is a multi-disciplinary open access archive for the deposit and dissemination of scientific research documents, whether they are published or not. The documents may come from teaching and research institutions in France or abroad, or from public or private research centers.
L'archive ouverte pluridisciplinaire HAL, est destinée au dépôt et à la diffusion de documents scientifiques de niveau recherche, publiés ou non, émanant des établissements d'enseignement et de recherche français ou étrangers, des laboratoires publics ou privés. 


\title{
Un gisement belloisien sur les bords de la Seine: le Closeau à Rueil-Malmaison (Hauts-de-Seine)
}

\begin{abstract}
Résumé
Le gisement du Closeau (Rueil-Malmaison, Hauts-de-Seine), situé à $5 \mathrm{~km}$ en aval de Paris, constitue une découverte fondamentale dans l'extension des recherches sur les périodes tardiglaciaires postérieures au Magdalénien. II permet notamment de documenter l'évolution des groupes aziliens dans le Bassin parisien. Au sud du gisement, un secteur constituant le sujet du présent article a livré une industrie tardiglaciaire différente, apparenté au Belloisien. L'étude typo-technologique de l'un des locus de ce secteur permet dans un premier temps de documenter les intentions et les modalités de la production lithique. Puis, la recherche d'éléments de comparaison tant techniques qu'économiques avec d'autres sites documentés offre la possibilité de confirmer l'appartenance de cette série au faciès Belloisien et de préciser l' identité culturelle" des tenants de cette tradition.
\end{abstract}

\begin{abstract}
Located $5 \mathrm{~km}$ downstream from Paris, Le Closeau (Rueil-Malmaison, Hautsde-Seine) is one of the most fundamental discoveries in recent research on postMagdalenian Late Glacial periods. It allows in particular the evolution of Azilian societies in the Paris Basin to be documented. In the southern part of the site, one sector yielded an original lithic industry attributable to the Belloisian. The typo-technological study presented here reveals the intentions and modes of lithic production. Then technical and economic comparisons with other sites confirm the attribution of the assemblage to the Belloisian facies and specify the "cultural identity" of the upholders of this tradition.
\end{abstract}

Avant la découverte du Closeau, les données sur les groupes humains du Tardiglaciaire postérieurs au Magdalénien dans le centre et le sud du Bassin parisien restaient parcellaires et délicates à interpréter (Bodu et Valentin, 1997). Les gisements connus étaient peu nombreux et surtout, ils manquaient essentiellement de données chronostratigraphiques. Depuis le début des années 90, sous l'impulsion du projet collectif de recherche Habitats et peuplements du Tardiglaciaire dans le Bassin parisien coordonné par M. Julien (Bodu et al., 1994), de nouveaux jalons sont posés et comparés notamment avec les recherches entreprises depuis les années 80 par J.-P. Fagnart et son équipe dans la vallée de la Somme (Fagnart, 1997a et b). C'est donc à cette époque "charnière" de la recherche que le site du Closeau, localisé sur la commune de Rueil-Malmaison (Hauts-de-Seine) est découvert en octobre 1994 sur le tracé de l'autoroute A 86. Situé dans le centre du Bassin parisien, à $5 \mathrm{~km}$ en aval de Paris, il est distant d'environ $300 \mathrm{~m}$ de la Seine et implanté sur sa rive gauche (fig. 1). Le Closeau se caractérise comme une occupation de fond de vallée, dominée par le Mont Valérien à l'est et les coteaux de la Jonchère au sud.

POSITION CHRONOLOGIQUE
ET STRATIGRAPHIQUE DES OCCUPATIONS
TARDIGLACIAIRES DU CLOSEAU
(d'après Bodu [dir.], 1998)

Outre l'importance de la surface fouillée (près de $25000 \mathrm{~m}^{2}$ ) et les possibilités d'interprétation palethnographique, l'un des intérêts majeurs du Closeau réside 


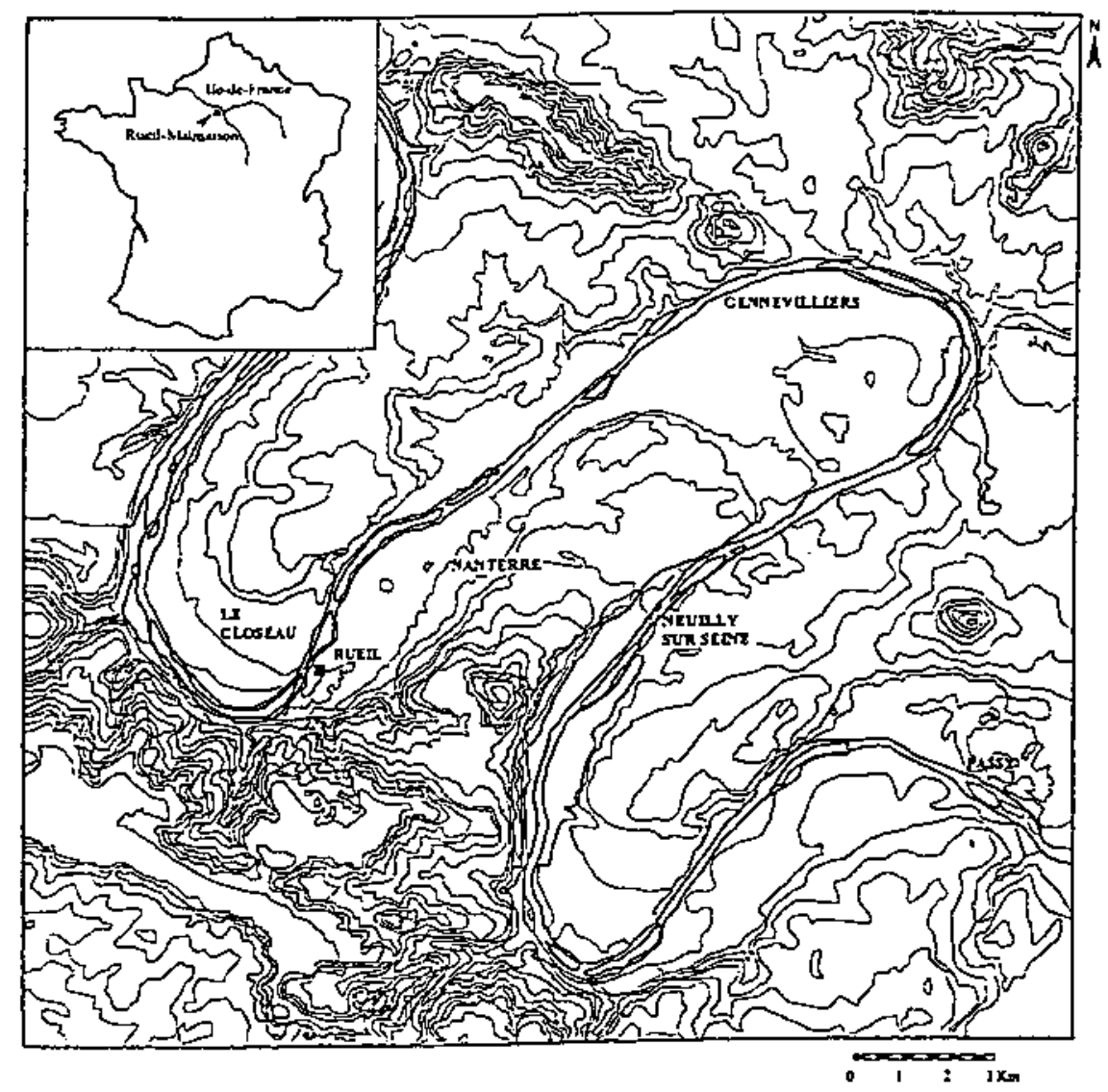

Fig. 1 - Localisation gćographique du Closenu (Ruei1-Malmaison, Flauts-de-Seine) (d'après Bodu [dir.], 1998).

dans la diachronie de la séquence témoignant de plusieurs moments d'évolution des groupes humains au Tardiglaciaire.

Le premier est repéré dans le niveau inférieur du paléochenal, localisé à la base du sol de l'Alleröd. Il livre deux datations ${ }^{14} \mathrm{C}$ confirmant les observations stratigraphiques' : $12090 \pm 90$ B.P. (cal. 12 511-11 846 B.C.) et $12050 \pm 100$ B.P. (cal. $12474-11785$ B.C.) Ce niveau est interprété comme un premier degré de l'azilianisation avec une économie des matières premières et des intentions de la production encore proches du Magdalénien supérieur local. La production lithique, tournée vers l'obtention de lames normalisées au profil rectiligne, est réalisée à partir d'une matière première locale soigneusement mise en forme. La percussion à la pierre tendre est exclusive tout au long de la chaîne opératoire et l'outillage voit la domination des grattoirs et l'unispécificité des armatures de type bipointes symétriques à dos courbe.

Le second est marqué par le niveau intermédiaire situé dans la seconde partie de l'Allerðd, entre 11300 et 11100 B.P. Le débitage laminaire de qualité, réalisé à la pierre tendre, n'atteint pas le niveau de mise en forme identifié dans le niveau inférieur. L'outillage se diversifie tant dans ses types avec présence de monopointes asymétriques que dans les supports utilisés.

Le troisième est rapporté à une phase tardive de l'Azilien, à la fin de l'Alleröd, voire au début du Dryas récent ( $10840 \pm 110$ B.P. ou cal. $11047-10568$ B.C.) Comparé aux deux niveaux précédents, le changement est radical avec absence de mise en forme et utilisation exclusive de la percussion directe dure. Il s'agit pour l'essentiel d'un débitage unipolaire d'éclats allongés tourné vers la confection d'un outillage peu diversifié composé essentiellement de pointes à dos courbe et de grattoirs.

Il faut également signaler la présence, dans le locus 25 , d'une industrie dominée par les pointes de Malaurie qui ne trouve que très peu d'équivalents parmi les autres secteurs du gisement. Les premières datations ${ }^{14} \mathrm{C}$ placent cet ensemble au tout début du Dryas récent (10 $755 \pm 90$ B.P. (cal. $10933-10516$ B.C.) et $10885 \pm 85$ B.P. (cal. 11 044-10 660 B.C.) Nous serions donc en présence d'un quatrième moment dans l'évolution des groupes aziliens. Ce constat est relativisé par l'incertitude reposant sur certaines datations absolues obtenues à partir de charbons de bois prélevés dans des structures de combustion dont l'origine anthropique reste encore discutée, au regard des probables phases d'incendies naturelles ayant affecté le gisement.

Enfin, un second secteur paléolithique, découvert au sud đu gisement et dénommé Sud R.N. 13, présente une industrie différente de celles rencontrées dans les différents niveaux aziliens du chenal. Les premières observations d'ensemble réalisées sur le matériel lithique indiquent que les populations installées en bas 
des coteaux de la Jonchère ne s'intègrent pas dans la même tradition technique que celles rencontrées dans le chenal. Le style du débitage et la composition de l'outillage incitent $\mathrm{P}$. Bodu à y voir un moment tardif du Tardiglaciaire : le Belloisien'.

\section{PRÉSENTATION GÉNÉRALE DE L'ÉTUDE : LES PROBLEMES, LES OBJECTIFS,} LES MÉTHODES

La découverte d'un second gisement paléolithique au Closeau, dénommé Sud R.N. 13 et actuellement appréhendé sur plus de $7000 \mathrm{~m}^{2}$, constitue une découverte originale dans le sud du Bassin parisien. Sur cette terrasse au pendage doux, la stratigraphie est comprimée, rendant parfois délicate la distinction des différents horizons archéologiques. Ce gisement livre également plusieurs niveaux mésolithiques et néolithiques et il convient de rester prudent quant à d'éventuelles contaminations inter-couches. Par ailleurs, la stratigraphie est moins complète que dans le chenal et elle ne permet pas de rattacher ce niveau paléolithique à la chronologie relative établie pour les différents moments de l'Azilien. De fait, les modalités de recouvrement du Sud R.N. 13 diffèrent de ce que nous connaissons dans le chenal. En raison de la topographie des lieux et de l'éloignement du fleuve, la phase sableuse caractérisant les dépôts du chenal n'est pas représentée ici et le recouvrement des pièces archéologiques, moins rapide, entraina vraisemblablement la destruction des restes osseux et laisse encore une épaisse patine blanchâtre sur les artefacts lithiques. P. Bodu note cependant que, "même si l'on doit écarter la possibilité d'une couverture rapide des vestiges paléolithiques par des alluvions de la Seine, on ne peut que constater le bon état général de conservation du second gisement du Closeau " (Bodu [dir.], 1998, p. 30).

L'objectif de cette étude est de détailler les modalités de production et de documenter selon un axe typotechnologique la composition de l'outillage : autrement dit, de faire ressortir les intentions sous-jacentes au débitage (Pelegrin, 1995 ; Valentin, 1995). L'ensemble de ces analyses est ensuite réuni afin d'apporter la documentation nécessaire pour établir des points de comparaison raisonnables entre le Sud R.N. 13 et d'autres séries. L'absence de cadre stratigraphique précis et de datations absolues impose pour l'heure de concentrer nos efforts sur le matériel lithique afin d'espérer replacer cet ensemble dans un cadre plus général. De ce fait, deux orientations principales guident cette étude : d'une part, il s'agit de documenter une partie du matériel lithique du Sud R.N. 13 selón une approche technologique orientée. Nous entendons par le terme "orienté" que nous recherchons les éléments diagnostiques de la série, au potentiel informatif le plus évident. Il ne s'agit donc pas d'une étude exhaustive des restes de taille mais d'une caractérisation des " moments-clés" de la production. D'autre part, nous utilisons ces informations dans le cadre d'une recherche bibliographique afin d'établir des points de comparaison entre l'assemblage étudié et d'autres séries déjà documentées. Ce n'est qu'à partir de ce moment que nous pourrons envisager la mise en évidence de relations unissant le secteur Sud R.N. 13 du Closeau et d'autres assemblages porteurs d'une tradition technique comparable.

En raison de l'extension spatiale (plus de $7000 \mathrm{~m}^{2}$ ) et quantitative (près de 20000 artefacts lithiques) de l'occupation paléolithique du Sud R.N. 13, notre attention s'est portée sur l'un des locus reconnus au préalable lors des opérations de terrain. Ce choix, défini selon des critères quantitatifs et qualitatifs en terme de composition de l'assemblage, s'est orienté vers le locus $P$ rassemblant environ 3000 artefacts lithiques et une proportion relativement importante, dans le cadre de ce gisement, de nucléus et d'outils. Nous ne nous sommes néanmoins jamais interdit d'aller rechercher des éléments dans les autres locus du gisement, notamment lors de l'étude de pièces jugées "diagnostiques" comme les pointes à dos.

Ce travail évolue dans un contexte analytique désormais bien déterminé et dont les principes méthodologiques ont fait l'objet de synthèses particulièrement précises (Pelegrin, 1995 ; Perlès, 1987 ; Valentin, 1995). Réalisée conjointement, l'évaluation des schémas opératoires de production et des intentions les guidant permet de replacer une série lithique à l'intérieur d'une tradition technique, de distinguer des différences et de mettre en avant des points récurrents entre plusieurs assemblages. C'est en développant de tels outils méthodologiques qu'il est dans certains cas possible de rattacher piusieurs ensembles non datés à un même "techno-complexe" ou de les rapprocher d'ensembles datés et positionnés à l'intérieur d'un cadre chronostratigraphique.

\section{ÉTUDE TYPO-TECHNOLOGIQUE DE L'INDUSTRIE LITHIQUE DU LOCUS P DU SECTEUR SUD R.N. $13^{3}$}

Le locus $P$ est localisé dans le quart sud-ouest du secteur Sud R.N. 13. Fouillé sur une surface d'environ $1000 \mathrm{~m}^{2}$, ce locus regroupe plusieurs concentrations de densité inégale et des zones vierges en vestige. Le matériel lithique, exclusif, se compose essentiellement de silex taillés, à l'exception de rares galets ou fragments de grès (tabl. 1). L'ensemble du matériel lithique

\begin{tabular}{|l|r|}
\hline Nucléus & 92 \\
\hline Outils & 270 \\
\hline Lames et fragments de lames & 47 \\
\hline Éclats et fragments (sup. à $3 \mathrm{~cm}$ ) & 1310 \\
\hline Éclats et fragments (inf. à 3 cm) & 787 \\
\hline Cassons & 312 \\
\hline Divers (galets, fragments de grès) & 21 \\
\hline Total & 2839 \\
\hline
\end{tabular}

Tabl. I - Le Closeau, secteur Sud R.N. 13 (locus P) : décompte de l'industrie recueillie. 
est recouvert d'une patine blanchâtre relativement épaisse ; en revanche, les tranchants ne sont pas détériorés et conservent une acuité correcte.

\section{Les objectifs du débitage: première approche}

Cette approche est rendue délicate par la rareté des pièces retouchées. Nous sommes dès lors amenés à considérer ici, outre les outils, l'ensemble de la production laminaire et les nucléus.

\section{Les principales caracténistiques des pièces retouchées}

Trois grands groupes typologiques représentés par les pièces à dos, les grattoirs et les denticulés dominent cette série (tabl. 2) et chacun d'eux témoigne d'une relative stabilité dans la conception de l'outillage.

\begin{tabular}{|l|c|c|c|}
\hline \multirow{2}{*}{ Types d'outlls } & \multicolumn{3}{c|}{ Supports } \\
\cline { 2 - 4 } & Lame & Éclat & Total \\
\hline Pointes à dos & 13 & & 13 \\
\hline Grattoirs & 4 & 8 & 12 \\
\hline Dentìulés & 1 & 7 & 8 \\
\hline Pièces à retouche marginale & 2 & 2 & 4 \\
\hline Burins & 2 & 1 & 3 \\
\hline Troncatures & 2 & & 2 \\
\hline Pièces mấchurées & 1 & 1 & 2 \\
\hline Lame retouchée & 1 & & 1 \\
\hline Couteau à dos & & 1 & 1 \\
\hline Fragment d'outil indéterminé & & 1 & 1 \\
\hline Total & 26 & 21 & 47 \\
\hline
\end{tabular}

Tabl. 2 - Le Closeau, secteur Sud R.N. 13 (locus P) : décompte de l'outillage retouché selon la nature du support.

Les pièces à dos comprennent majoritairement des pointes à dos auxquelles il convient d'ajouter un couteau à dos (fig. $\left.2, \mathrm{n}^{\circ} 1\right)$. Le groupe des pointes à dos
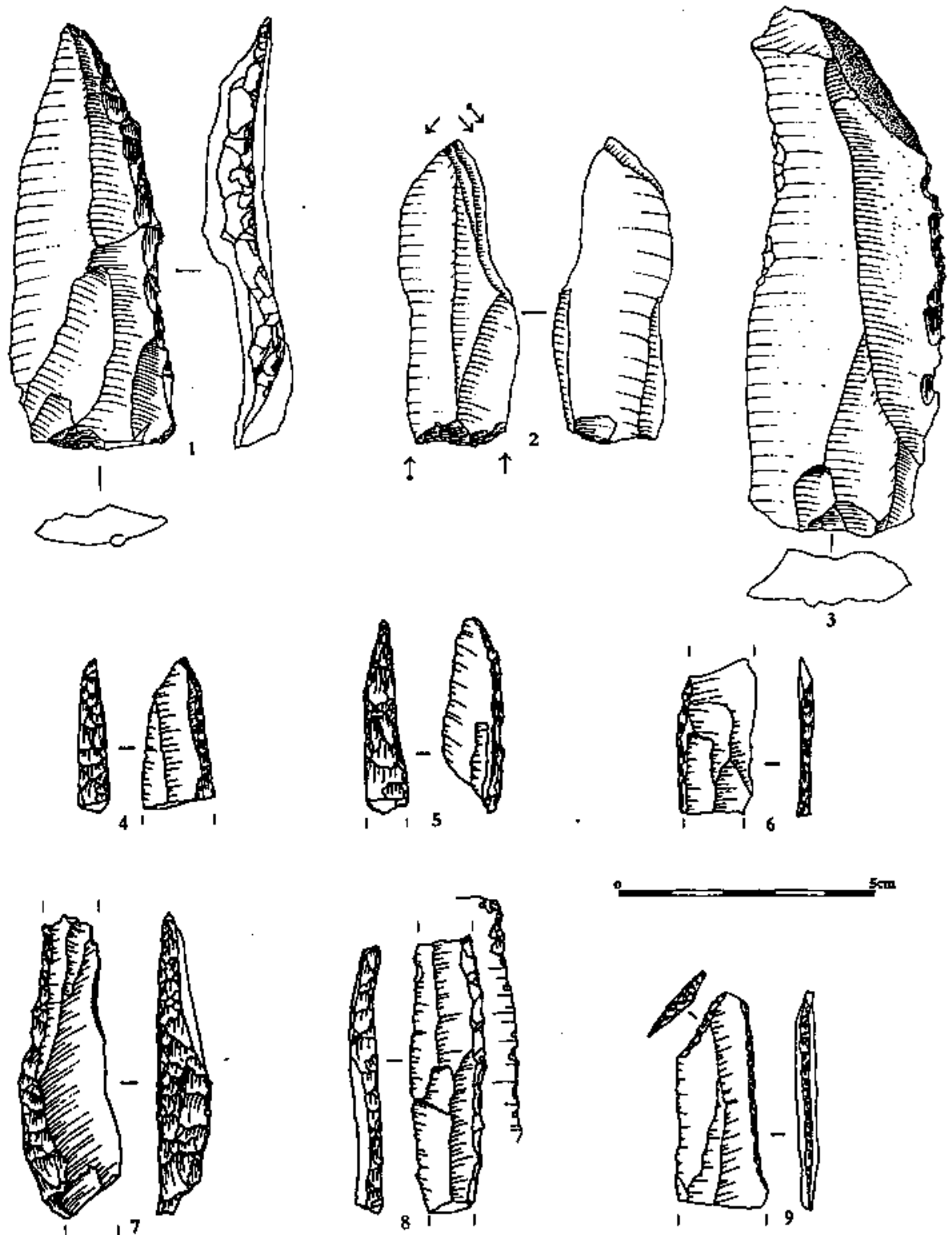

Fig. 2 - Le Cioseau, secteur Sud R.N. 13. Outillage : 1 : couteau a dos ; 2 : burin multiple mixte ; 3 : denticulè; 4 à 9 : pointes à dos (dessins : 1 a $3:$ N. Teyssandier; 4 à 9 ; F. Kildéa). 

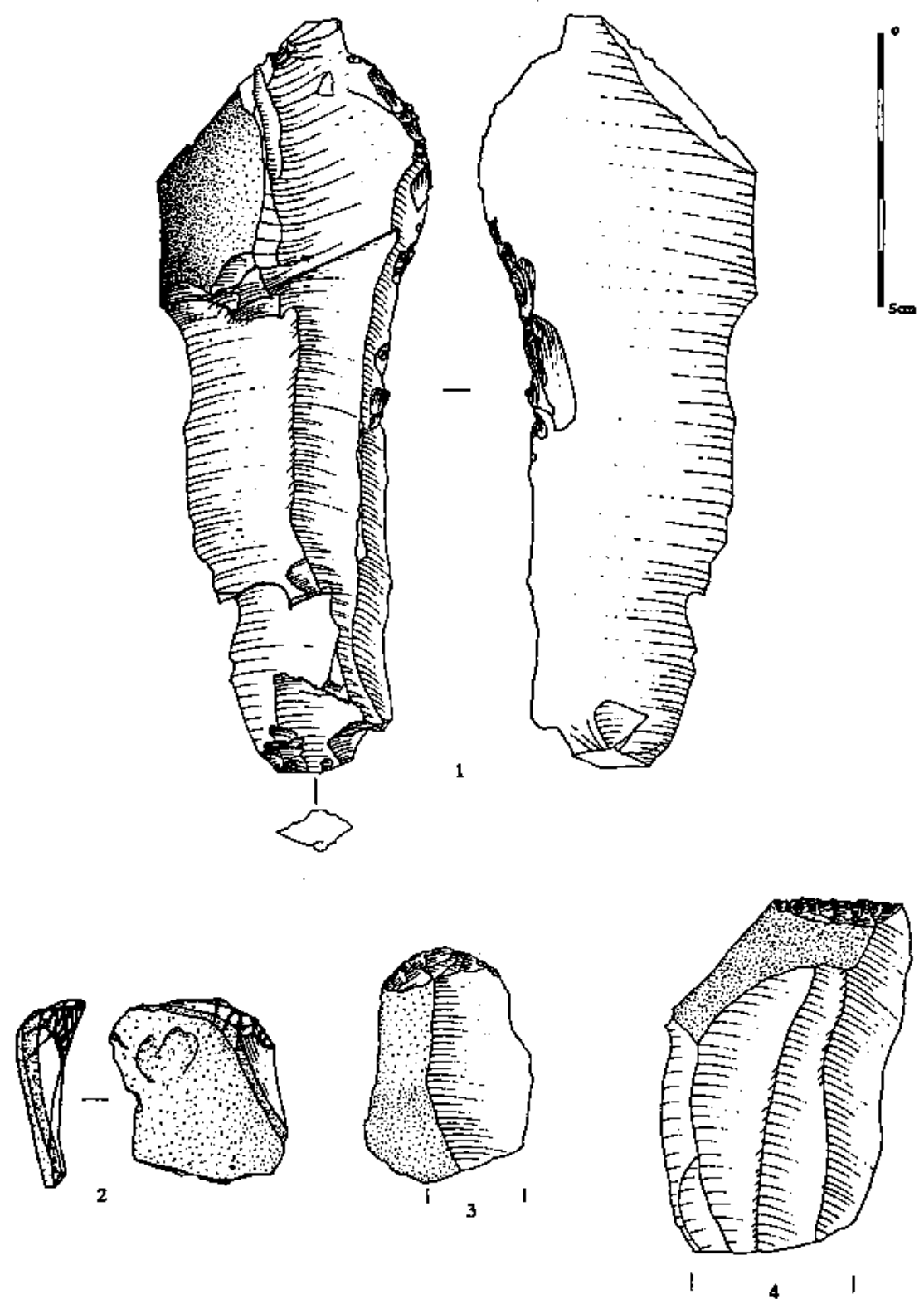

Fig. 3 - Le Closeau, secteur Sud R.N. 13. Outillage : 1 : lame mâchurée; 2 et 3 : grattoirs simples; 4 : pièce à troncature rectiligne (dessins : 1,3 et $4: \mathrm{N}$. Teyssandier; $2: \mathrm{P}$. Alix).

(fig. $2, \mathrm{n}^{\circ \mathrm{s}} 4$ à 9 ) est composé de treize éléments fragmentés. Il est dominé par les pointes à dos rectiligne (cinq à dos épais, entre 4 et $7 \mathrm{~mm}$, et deux à dos mince de $2 \mathrm{~mm}$ environ) et dans une moindre mesure par les pointes à dos courbe. Le dos, toujours abrupt, est le plus souvent aménagé par une retouche directe, plus rarement croisée. Systématiquement aménagées sur lames, les pointes à dos présentent des dimensions relativement homogènes après retouchè, d'une largeur variant entre 8 et $14 \mathrm{~mm}$ et d'une épaisseur située entre 2 et $5 \mathrm{~mm}$. Ce sont toujours des petites lames de plein débitage assez régulières; leur profil est, dans la partie retouchée, fréquemment rectiligne. Quelques pointes à dos minces $(1$ à $2 \mathrm{~mm})$ indiquent que la retouche ne transforme parfois que très peu le support originel dont l'allure est déjà très élancée.
Réalisés tant sur éclats que sur lames, les grattoirs sont systématiquement confectionnés sur des supports de petites dimensions, généralement de médiocre régularité et partiellement corticaux (fig. 3, $\mathrm{n}^{\text {os }} 2-3$ ). Ils appartiennent tous au type des grattoirs simples et présentent un front relativement large aménagé par une retouche courte et semi-abrupte.

Les denticulés sont presque toujours réalisés sur éclat et se caractérisent par la sélection de sous-produits du débitage laminaire (fig. $2, n^{\circ} 3$ ). En fait, la morphologie du support importe peu et la seule présence d'un bord adéquat pour l'aménagement de plusieurs coches contiguës est suffisante. Il en est de même pour les pièces à retouche marginale et pour les pièces tronquées (fig. $3, n^{\circ} 4$ ). 
Le reste de l'outillage se partage en plusieurs types d'outils, quantitativement plus anecdotiques. Le contexte de cette série nous amène à nous attarder simplement sur la présence de deux pièces mâchurées (fig. $3, n^{\circ} 1$ ). Elles sont comparables aux " outils a posterior " précisément décrits dans certains ensembles du Paléolithique final du nord de la France et du sud-est de l'Angleterre (Barton et Roberts, 1997; Bodu et Valentin, 1992, 1993 ; Fagnart, 1997a et b ; Fagnart et Plisson, 1997). Les premières analyses expérimentales et tracéologiques en contexte Tardiglaciaire montrent que ces pièces ont pu servir à l'aménagement et l'entretien des percuteurs de pierre tendre (Fagnart et Plisson, 1997).

La prise en compte de l'outillage retouché met tout d'abord en évidence l'aspect dominant voire exclusif du débitage laminaire puisque tous les outils sont aménagés sur des lames ou des éclats issus de l'exploitation ou l'aménagement de nucléus à vocation laminaire. Parmi ces produits allongés, nous pouvons déjà isoler un groupe de petites lames étroites, de profil régulier, destiné à la confection des pointes à dos. En considérant la partie dı support éliminée lors de l'aménagement du dos, nous pouvons en déduire que les supports utilisés devaient approximativement être larges de 15 à $20 \mathrm{~mm}$ environ.

Enfin, une sélection systématique des sous-produits du débitage laminaire est observable pour les grattoirs et les denticulés avec notamment le choix de supports courts et corticaux pour les grattoirs.

\section{L'apport des nucléus et des supports laminaires bruts dans la caractérisation des objectifs de la production}

Parmi les 92 nucléus que compte la série, nos observations pour la recherche des intentions de la production se sont portées sur 50 pièces dont les derniers négatifs témoignent indubitablement du caractère laminaire de la production jusque dans ses derniers moments. Les 42 autres nucléus se décomposent en 21 fragments de nucléus indéterminés affectés par des altêrations thermiques, 3 préformes de nucléus à lames et 18 nucléus finissant en nucléus à éclats. Concernant ces derniers, il s'agit pour la plupart de nucléus à lames dont le débitage a été poursuivi jusqu'à l'obtention de quelques éclats.

Le critère distinctif le plus pertinent des nucléus laminaires semble correspondre à la largeur des derniers négatifs d'enlèvement réussis. Il a ainsi été possible de subdiviser ces pièces en cinq groupes (tabl. 3).
Au regard des dimensions des derniers négatifs réussis, trois groupes peuvent être distingués. Le premier (catégorie 1), très minoritaire, est composé de nucléus tournés vers l'obtention de lames larges et peu régulières; le second (catégorie 2 et 3 ) de nucléus à lames de largeur moyenne, globalement assez régulières et le troisième (catégorie 4 et 5) de nucléus à petites lames minces ou lamelles, très régulières.

L'examen des produits laminaires bruts confirme en partie la tripartition opérée à partir des nucléus. La série comprend 88 lames entières dont 22 sont rapportables à des séquences de plejn débitage 4 et 66 à des phases d'aménagement ou d'entretien.

Parmi les éléments de plein débitage, on note une prédominance des lames comprises entre 60 et $100 \mathrm{~mm}$ de Iong pour une largeur oscillant entre 15 et $25 \mathrm{~mm}$. Cette première information confirme nos premières impressions issues de l'analyse des nucléus montrant que le débitage est principalement orienté vers l'obtention de lames longues d'environ 50 à $90 \mathrm{~mm}$ et larges de 15 à $25 / 30 \mathrm{~mm}$. Ces supports laminaires sont le plus souvent réguliers et de profil plus ou moins rectiligne. Une observation fine des lames entières de plein débitage permet en partie d'expliquer leur abandon à l'état brut sur le gisement : elles comportent le plus souvent une légère irrégularité des bords ou du profil, parfois torse (fig. $4, \mathrm{n}^{05} 2,4$ et 5). La concentration de pièces entre 15 et $20 \mathrm{~mm}$ de large mise en évidence tant à partir des négatifs réussis sur les nucléus que sur les produits laminaires de plein débitage abandonnés peut s'expliquer comme la production de supports pour la confection des pointes à dos. Nous rappelons en effet qu'après l'aménagement du dos, les pointes mesurent entre 8 et $14 \mathrm{~mm}$ de large. Il est donc vraisemblable que parmi les lames larges de 15 à $20 \mathrm{~mm}$ environ, les plus régulières et rectilignes aient été sélectionnées pour la fabrication des pointes à dos.

Sur le locus P du Sud R.N. 13, la production de lames régulières et de profil assez rectiligne est le principal objectif des tailleurs. On peut aisément distinguer la recherche de modules distincts avec des lames larges et peu régulières, des lames plus étroites et plus régulières majoritaires et des petites lames minces ou des lamelles, très régulières et normalisées.

En fin de course, certains nucléus ont eu pour vocation de produire quelques éclats courts et larges, supports potentiels de certains grattoirs. Une grande partie de la production utilitaire semble avoir été produite sur place pour un usage différé. En témoignent la faible quantité d'outils et le petit nombre de lames de première intention recueillis.

\begin{tabular}{|c|c|c|c|c|c|}
\hline & Catégorie 1 & Catégorie 2 & Catégorie 3 & Catégorie 4 & Catégorie 5 \\
\hline \multirow{3}{*}{$\begin{array}{l}\text { Type de } \\
\text { nucléus } \\
\text { á lames }\end{array}$} & $\begin{array}{c}\text { Nucléus } \\
\text { à lames larges }\end{array}$ & $\begin{array}{l}\text { Nucléus à lames } \\
\text { de lacgeur moyenne }\end{array}$ & $\begin{array}{l}\text { Nucléus à lames } \\
\text { de latgeur moyenne }\end{array}$ & $\begin{array}{c}\text { Nucléus à petites lames } \\
\text { minces ou lamelles }\end{array}$ & $\begin{array}{l}\text { Nucléus à petites lames } \\
\text { minces ou lamelles }\end{array}$ \\
\hline & (sup. a $3 \mathrm{~mm}$ ) & $(15$ a $30 \mathrm{~mm})$ & $(15$ à $30 \mathrm{~mm})$ & $(7$ a $15 \mathrm{~mm})$ & $(7$ à $15 \mathrm{~mm})$ \\
\hline & Exploitation unipolaire & Exploitation unipolaire & Exploitation bipolaire & Exploitation unipolaire & Exploitation bipolaire \\
\hline Effectif & 3 & 20 & 9 & 13 & 5 \\
\hline
\end{tabular}

Tabl. 3 - Types de nucléus laminaires selon le module de largeur des derriers négatifs d'enlèvement réusssis et le caractère uni ou bipolaire du débitage. 

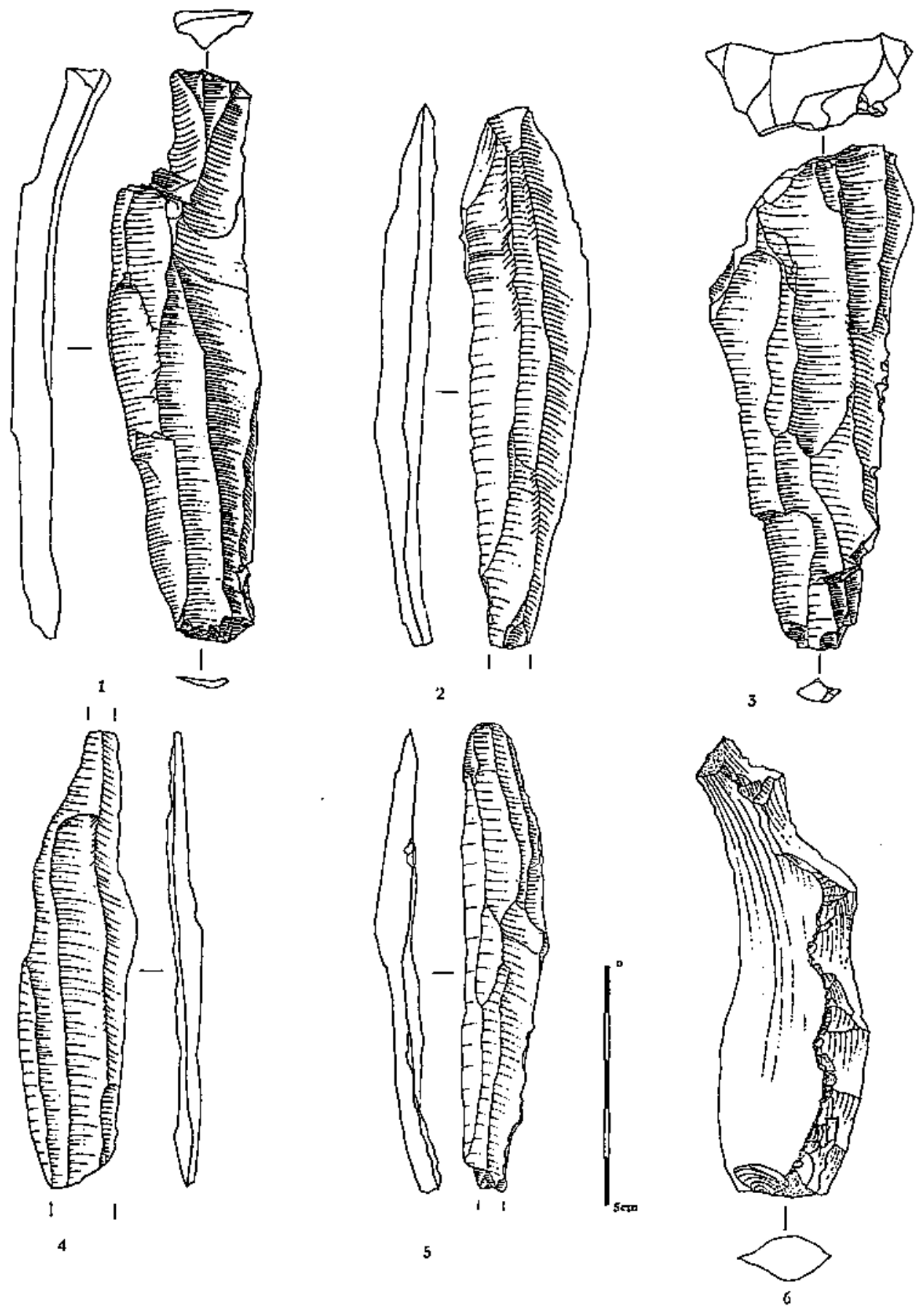

Fig. 4 - Le Closeau, secteur Sud R.N. 13 : Lames brutes : 1 et 3 : lames outrepassées emportant le plan de frappe opposé ; 2, 4 et 5 : lames de plein débitage ; 6 : lame à crête à un versant (dessins : N. Teyssandier).

\section{Les matières premières débitées}

La totalité des produits que contient la série a été débitée dans deux matériaux d'origine locale. Le silex secondaire constitue l'essentiel des matériaux utilisés par les occupants du Sud R.N. 13, soit plus de $99 \%$ du matériel lithique. Cette matière première est présente à l'aplomb du site, à moins de $50 \mathrm{~m}$, dans les bancs du Campanien mis en évidence sur les coteaux de la Jonchère (Bodu [dir.], 1998). Il s'agit d'un silex de bonne qualité, au grain fin à moyen, présentant parfois des zones plus grenues et des plans de fracture n'ôtant pas son homogénéité générale, le rendant parfaitement apte au débitage laminaire. Les prospections réalisées dans cette zone montrent que ces silex sont abondants, de toutes dimensions et d'une morphologie allongée et globalement assez régulière. Les états de surface relevés sur le matériel archéologique indiquent la nette prédominance des surfaces corticales légèrement lessivées mais non roulées. L'acquisition pouvait ainsi se faire en contrebas des bancs de silex campanien, en position secondaire toute proche, les blocs ayant été préalablement déchaussés de leur substratum. Quelques blocs seulement présentent des surfaces d'altération 
caractéristiques d'un approvisionnement dans les alluvions de la Seine. En termes dimensionnels, les paléolithiques se sont concentrés sur les blocs de moyennes ou petites dimensions, entre 10 et $25 \mathrm{~cm}$ environ.

Le silex tertiaire représente une faible part des matériaux utilisés sur le site. Nous avons néanmoins pu observer la présence de deux nucléus et de quelques produits de débitage témoignant du traitement de ce matériau sur le site. D'origine locale, le silex tertiaire est présent dans la strate dite "calcaire de Champigny" de l'étage Marinésien inférieur bordant les reliefs de la Jonchère (Bodu [dir.], 1998). Il se rencontre sous la forme de plaquettes, d'épaisseur variable et de qualité moyenne.

L'examen des produits débités plaide pour une arrivée sur le site de blocs bruts ou tout juste testés ; les éclats d'entame sont relativement nombreux et parfois de grandes dimensions. Tous les produits intermédiaires de mise en forme et d'entretien sont présents, attestant du déroulement complet de la chaîne opératoire sur le gisement. Enfin, les éclats de mise en forme dont les dimensions sont parfois conséquentes (plus de $15 \mathrm{~cm}$ de long dans certains cas) indiquent une exploitation de nodules assez volumineux, en contraste avec la petite taille de la majorité des nucléus et renseignant donc sur un état d'exhaustion conséquent de ceux-ci. À ce propos, une observation fine de certains petits nucléus témoigne de l'introduction sur le site de volumes dimensionnellement variés, vraisemblablement en rapport avec les objectifs de la production.

\section{Traitement des matériaux en vue du débitage laminaire}

Concernant le silex tertiaire, nous serons d'autant plus bref qu'il est très largement anecdotique dans cette série. Seuls deux nucléus, restés à l'état d'ébauches de nucléus à lames, montrent une mise en forme par le jeu d'une crête postéro-médiane à deux versants préparés. Les renseignements les plus précieux sont bien évidemment fournis par le traitement des volumes en silex secondaire. Plusieurs modalités techniques de mise en forme ont été rencontrées et décryptées indirectement par l'examen de certains produits caractéristiques. En l'absence de "remontages physiques", notre description reste relativement synthétique.

La plus simple expression des modalités de mise en forme se retrouve sur des volumes pour lesquels cette séquence est rédıite à l'ouverture d'un plan de frappe et l'extraction d'une lame d'entame corticale. C'est dans ce cas l'utilisation d'une morphologie initiale qui caractérise les premiers moments du débitage. Il est également fréquent que lors de la poursuite de l'exploitation, le dos et les flancs des nucléus restent corticaux (fig. $5, n^{\circ} 2$ ). Cependant, la mise en évidence de nucléus conservant des surfaces naturelles tout au long du débitage n'implique pas forcément que ces volumes n'aient pas fait l'objet d'une mise en forme. Bien souvent et suivant l'ampleur des séquences laminaires, les traces d'aménagement de la surface de débitage peuvent avoir été effacées. Par exemple, seuls quatre nucléus ont conservé des portions de négatifs de la mise en place d'une crête d'entame. Pourtant, parmi les restes de taille, nous avons identifié 32 lames à crêtes ou souscrêtes. Le plus souvent, il s'agit de crêtes totales à un ou deux versants préparés, đe profil peu courbe et détachées par percussion directe dure portée relativement à l'intérieur du plan de frappe (fig. $4, n^{\circ} 6$ ).

Lorsque le dos et les flancs sont aménagés, ils ne le sont que partiellement avec généralement la mise en place d'une crête antéro ou postéro-latérale. Rares sont les volumes aménagés plus globalement par le jeu de plusieurs crêtes puisque seuls cinq nucléus associent deux crêtes.

En résumé, il est donc relativement fréquent que les tailleurs n'effectuent qu'une courte mise en forme du volume à débiter avec ouverture d'un plan de frappe et extraction directe des premiers produits laminaires qui concourent alors à régulariser la surface de débitage. Toutefois, nous voulons ici insister sur le fait que dans bien des cas, l'aménagement du volume a pu être plus conséquent que ce que les nucléus ou même certaines "pièces techniques" laissent présager. Les premiers remontages effectués indiquent qu'un nombre important d'éclats de mise en forme peut être détaché avant l'exploitation du volume à des fins laminaires. Ả ce propos, nous avons globalement constaté deux types, morphologiquement đistincts, d'enlèvements de mise en forme : le premier constitué d'éclats épais et volumineux traduit une première phase de dégrossissage des blocs; le second se distingue par la moindre épaisseur des produits et leur aspect plus couvrant. Certains de ces éclats indiquent d'ailleurs un changement dans la gestuelle de percussion qui, d'une percussion rentrante à l'intérieur du plan de frappe devient tangentielle, le percuteur de pierre (tendre ?) venant accrocher le bord du plan de frappe, préalablement préparé par abrasion (Pelegrin, sous-presse). Ces produits sont interprétés comme les témoins d'une séquence visant à régulariser ou à entretenir les convexités des nucléus.

\section{L'exploitation laminaire}

L'exploitation laminaire débute donc par le détachement d'une lame d'entame corticale ou d'une lame à crête. Puis, le détachement des premières lames concourt à régulariser la morphologie de la surface de débitage.

Les séquences de plein débitage souffrent d'un manque de documentation en raison du faible nombre de produits laminaires entiers s'y rattachant. Cette carence s'explique en partie, semble-t-il, par le prélèvement des meilleurs supports pour un usage différé. Au vu des nucléus et surtout des produits caractéristiques du débitage, il semble que le plein débitage se déroule à partir d'une surface laminaire faiblement carénée (profil des lames de première intention très souvent rectiligne) et au cintre relativement ouvert, la surface laminaire initiale étant le plus souvent positionnée sur l'une des faces larges du bloc. Au cours de cette séquence, l'usage de la percussion à la pierre tendre est exclusif. Ce délicat diagnostic a pu être réalisé sur certains produits seulement ${ }^{5}$ présentant en association des stigmates caractéristiques: talons très minces 

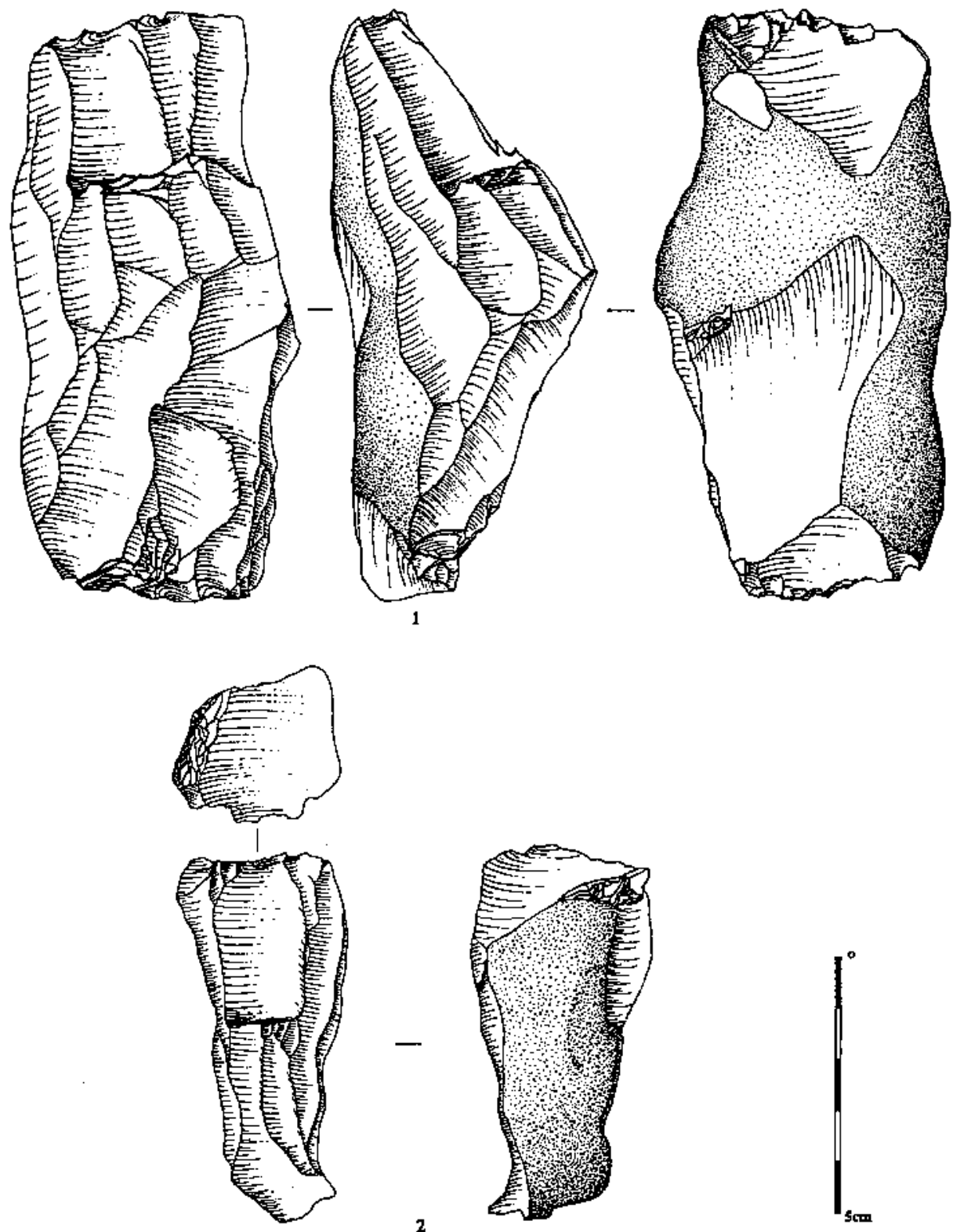

Fig. 5 - Le Closeau, secteur sud R.N. 13 : Nucléus à lames : 1 : nucléus bipolaire ; 2 ; nucléus unipolaire (dessins : N. Teyssandier).

parfois punctiformes, profil rectiligne des produits de première intention et abrasion systématique de la zone d'impact, esquillement très fréquent du bulbe, ondulations fines et serrées sur la face d'éclatement (Pelegrin, sous-presse avec nombreuses références documentées). Il est par ailleurs essentiel de retenir que les lames de plein débitage sont détachées selon une gestuelle tangentielle et non rentrante, et que leur extraction est assurée par une préparation préalable consistant en une abrasion et parfois un véritable doucissage de la zone d'impact.

Le débitage laminaire opère selon un schéma unipolaire (fig. $5, \mathrm{n}^{\circ} 2$; fig. $6, \mathrm{n}^{\circ} 1$ ) ou bipolaire (fig. $5, \mathrm{n}^{\circ} 1$; fig. $\left.6, \mathrm{n}^{\circ} 2\right)$. L'unipolarité du débitage paraît dominante puisque 36 des 50 nucléus à lames s'insèrent dans cette catégorie. Nous devons également prendre en considération le fait que certains nucléus unipolaires aient pu précédemment être exploités à partir đe deux plans de frappe dont l'un a pu disparaître à la suite d'un outrepassage massif (fig. $4, n^{\circ} 3$ ).

Lorsque le débitage est bipolaire, la mise en place des deux plans de frappe se déroule au début de l'exploitation laminaire. Ceci se vérifie par exemple sur une grande lame sous-crête outrepassée ayant emporté une partie du plan de frappe opposé et attestant que dès le détachement de la crête, les deux plans de frappe étaient déjà installés. Les nucléus et certains produits laminaires réguliers indiquent des changements d'orientation relativement rapides depuis les deux plans de frappe opposés. Pour les 22 lames entières de plein débitage, douze portent des négatifs de sens opposé qui dans la plupart des cas envahissent plus des deux-tiers de la face supérieure (fig. $4, \mathrm{n}^{\text {os }} 4-5$ ). De la même façon, les nucléus sur lesquels cette observation 

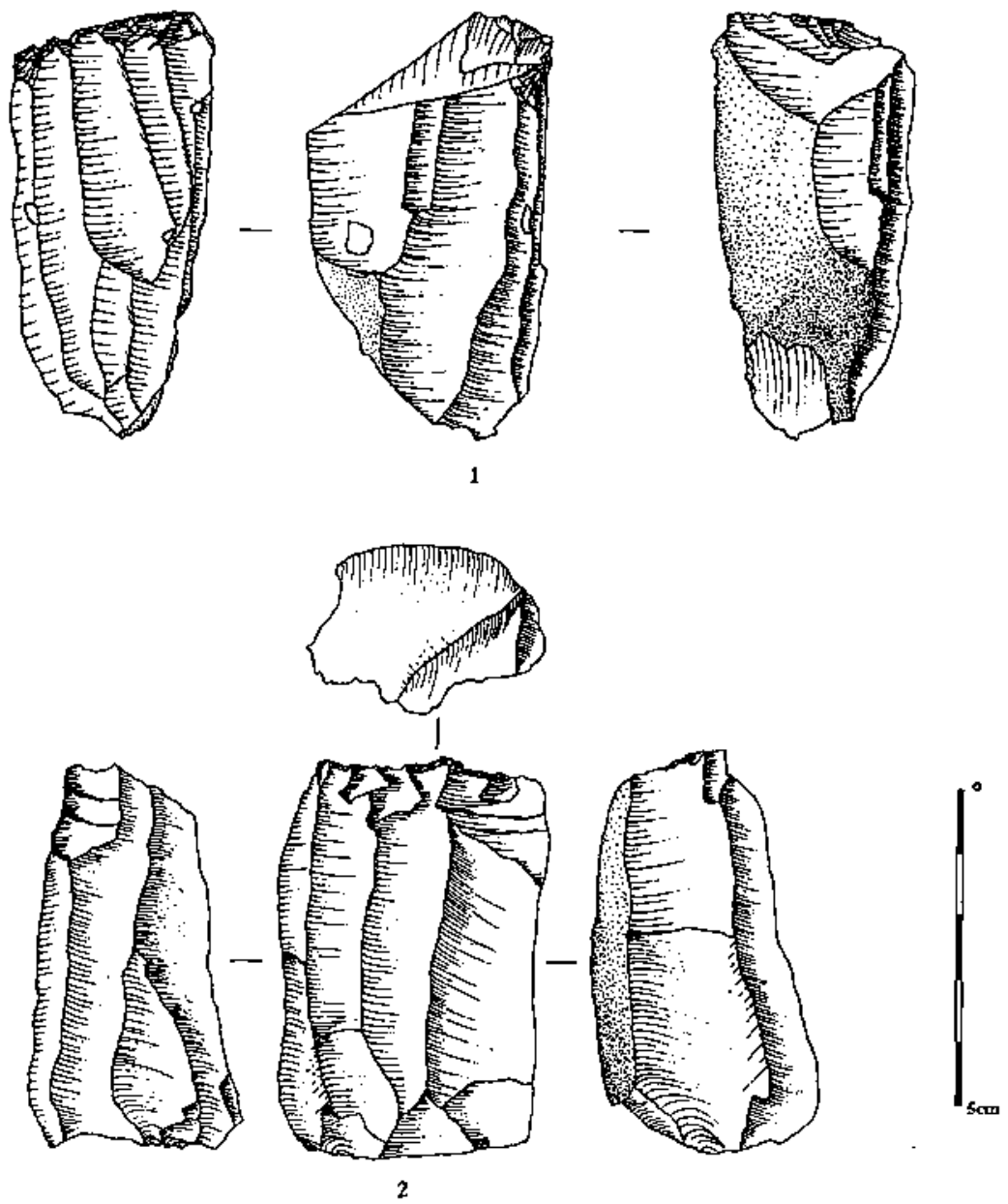

Fig. 6 - Le Closeau, secteur Sud R.N, 13 : Nucléus à lames : 1 : nucléus unipolaire; 2 : nucléus bipolaire (dessins: $\mathrm{N}$. Teyssandier).

est envisageable témoignent de plusieurs changements dans la polarité du débitage. Ainsi, nous pensons pouvoir affirmer que lorsque les tailleurs' s'orientent vers un débitage bipolaire, les produits laminaires de première intention sont extraits successivement à partir des deux plans de frappe selon une alternance vraisemblablement assez rapide. Ce choix permet de conserver une surface laminaire faiblement carénée, permettant l'obtention de lames au profil rectiligne. Mais cette modalité d'utilisation des plans de frappe n'est pas unique et dans certains cas, chaque plan de frappe est utilisé successivement de façon à fournir une série de lames. Ce rythme d'alternance plus lent aboutit fréquemment à des réflèchissements alors corrigés par des enlèvements nécessairement épais.

L'inclinaison des plans de frappe conserve toujours une obliquité assez importante, en rapport avec le caractère tangentiel de la production. En cours de débitage, les tailleurs agissent le plus souvent à partir de plans de frappe conservés lisses, cependant fréquemment renforcés par abrasion vers la surface laminaire ${ }^{6}$. Occasionnellement, lorsque le débitage ne peut plus être poursuivi, une tablette de ravivage est extraite depuis l'un des flancs du nucléus.
Le plus souvent, l'exploitation laminaire évolue à partir de la surface de débitage initiale et y reste concentrée. Le rythme du débitage est alors frontal, à partir d'une surface laminaire située sur l'une des faces larges du volume et dans son axe d'allongement le plus important. C'est notamment le cas des exploitations unipolaires pour lesquelles il est rare que le débitage envahisse l'un des flancs. Seuls quelques nucléus unipolaires, apparemment les plus productifs, témoignent d'une modalité d'évolution du débitage selon un rythme particulier. Sur ces volumes, le débitage envahit successivement plusieurs surfaces sécantes. Cette modalité permet en effet la poursuite du débitage sur des surfaces naturellement cintrées par le rythme de détachement des supports laminaires. Concernant les nucléus de schéma bipolaire et essentiellement pour ceux produisant de petites lames, le débitage évolue selon un rythme semi-tournant, envahissant au moins l'un des flancs (fig. $6, \boldsymbol{n}^{\circ} 2$ ).

Dans tous les cas, et ce quelles que soient les intentions de la production, le débitage est toujours interrompu suite à des accidents liés à des problèmes de convexité (fig. 5 et 6). Les tailleurs évoluant sur des surfaces faiblement carénées rencontrent au fur et à mesure de 
l'évolution du débitage des difficultés à contrôler cette convexité longitudinale, sa diminution progressive aboutissant à des réflèchissements systématiques. Dans certains cas, et notamment sur les petits nucléus, la poursuite đu débitage devient impossible. L'exploitation đe quelques nucléus de plus grandes dimensions aurait pu être poursuivie mais les réaménagements nécessaires demandaient une réorientation des objectifs de la production. Enfin, sur quelques nucléus, le débitage se poursuit et vise l'obtention d'éclats courts et larges détachés par percussion directe dure.

\section{ÉLÉMENTS DE COMPARAISON : VERS UNE IDENTIFICATION CHRONO-CULTURELLE DES OCCUPANTS DU SITE?}

Les grandes modalités techniques de la production lithique du secteur Sud R.N. 13 du Closeau étant connues, il reste à les replacer dans un contexte plus large afin de trouver, si cela est possible, tes points de comparaison entre cette série et d'autres gisements. En effet, cette série présente la particularité de n'avoir livré aucune information la rattachant à une chronologie, qu'elle soit absolue ou relative ${ }^{7}$. Seul l'examen du matériel archéologique, nécessairement lithique étant donné les conditions taphonomiques, permet de documenter celte recherche. Nous limiterons volontairement cette recherche comparative à la période tardiglaciaire, Magdalénien non compris (de la transition Bölling/Alleröd jusqu'au Préboréal), compte-tenu des profondes différences entre l'industrie étudiée et la production lithique magdalénienne identifiée dans les gisements d'Ile-de-France (Audouze et al., 1988). Pour les autres techno-complexes du Paléolithique supérieur, les comparaisons ne sont d'ailleurs pas plus probantes. Pour l'ensemble de ces raisons, auxquelles nous ajouterons la spécificité du Closeau en terme chronologique ${ }^{8}$, nous étendrons uniquement cette recherche à d'autres industries de la fin du Tardiglaciaire. Sur le gisement du Closeau, le Tardiglaciaire est représenté par plusieurs horizons successifs. Les trois principaux moments de l'Azilien distingués par P. Bodu (niveau inférieur, niveau intermédiaire et niveau supérieur) n'offrent pas de points de convergence convaincants avec le secteur Sud R.N. 13.

Pour le niveau inférieur, les armatures sont différentes (bipointes symétriques à dos courbe dont le dos est souvent constitué d'une retouche marginale) tout comme la proportion de l'outillage. Ainsi, la part de l'outillage atteint 14 à $18 \%$ dans les locus du niveau inférieur alors qu'elle dépasse rarement 2 à $3 \%$ dans les locus du Sud R.N. 13.

Pour le niveau intermédiaire, l'outillage est également plus abondant ( $6 \%$ et plus) que dans les locus du Sud R.N. 13 et surtout plus diversifié. Les armatures se composent essentiellement de monopointes asymétriques dont les supports apparaissent moins standardisés que ceux du Sud R.N. 13. Le débitage laminaire, de qualité, est moins sophistiqué, notamment dans la préparation au détachement des lames comme dans les modalités de mise en forme.

Enfin, le niveau supérieur de l'Azilien est certainement le plus facile à distinguer du secteur Sud R.N. 13. Les données technologiques montrent un débitage peu organisé avec une mise en forme courte voire totalement absente et l'utilisation de la percussion directe dure portée en profondeur du plan de frappe tout au long de la chaîne opératoire. Cette exploitation fournit en majorité des éclats laminaires et plus rarement des lames peu normalisées. De la même façon, les armatures (monopointes asymétriques à dos courbe) sont aménagées sur des supports pas toujours réguliers, parfois même sur des éclats.

Pour résumer, aucune des trois grandes tendances de l'azilianisation reconnues au Closeau (Bodu [dir.], 1998 ; Bodu et Valentin, 1997) ne concordent avec les données recueillies sur le secteur du Sud R.N. 13. Des discordances typologiques, technologiques et même économiques stigmatisent des comportements distincts. Nous serions donc ici en présence d'un autre moment d'évolution des industries tardiglaciaires en Ile-de-France. En fait, au sein de la séquence du Closeau, seul le niveau à pointes de Malaurie (locus 25) peut-être apparenté aux traditions d'affinités laboriennes, rappelle par certains éléments l'industrie du Sud R.N. 13. Cet horizon, placé à l'extrême fin du Dryas récent par des datations sur charbons de bois $(10755 \pm 90$ B.P. et $10885 \pm 85$ B.P.) livre un matériel archéologique peu abondant mais suffisamment cxplicite pour qu'il soit intéressant de s'y attarder. Ce sont surtout les pointes à dos qui dénotent avec le reste de la séquence tardiglaciaire: pour l'essentiel, il s'agit de véritables pointes de Malaurie (pointes à dos très rectiligne et base tronquée) aménagées sur des supports très standardisés et réguliers. Elies sont réalisées sur un silex blond translucide à grain fin qui n'a pas d'équivalent dans les débitages reconnus sur ce locus. Ces derniers attestent par ailleurs d'une phase de mise en forme relativement soignée mais n'apportent que peu de renseignements sur le plein débitage, les produits de première intention étant absents. Ces quelques éléments permettent de dresser les premières liaisons entre le secteur Sud R.N. 13 et un autre locus du gisement du Cioseau situé dans la zone du chenal principal. Néanmoins, ces rapprochements doivent pour l'heure être pris avec grande précaution et nous les reconsidérerons avec plus d'attention, lors de nos comparaisons inter-sites et extra-régionales.

L'élargissement extra-régional des comparaisons avec d'autres gisements rattachés aux groupes à Federmesser et aziliens ne livre pas non plus d'éléments de comparaison convaincants (Fagnart, 1997a et b; Valentin, 1995). Schématiquement, J.-P. Fagnart caractérise ces industries à Federmesser par "la mise en forme des blocs, très réduite, (qui) vise à l'extraction de lames courtes, peu calibrées et souvent irrégulières obtenues généralement au percuteur de pierre " (Fagnart, 1997b, p. 60). Finalement, pendant toute la partie du Tardiglaciaire comprise entre la transition Bölling/Alleröd et la fin de la chrono-zone de l'Alleröd, période de développement des groupes aziliens, nous ne retrouvons pas de 
lien de parenté particulier avec l'assemblage ici étudié. En revanche, et comme nous le détaillerons désormais, à partir du Dryas récent et peut-être plus encore lors du Préboréal, on assiste à un changement important dans les traditions techniques qui nous semblent alors plus proches de ce que nous retrouvons sur le locus $P \mathrm{du}$ Sud R.N. 13.

\section{LA PROBABLE PRÉSENCE AU CLOSEAU} D'INDUSTRIES BELLOISIENNES

En effet, c'est avec les industries dites "belloisiennes" que nous retrouvons le plus d'affinités. Celles-ci sont pour l'instant uniquement connues sur "des sites spécialisés dans l'acquisition et le débitage très soigneux de silex d'excellente qualité " (Bodu et Valentin, 1997, p. 345). Il s'agit donc d'un ensemble de séries lithiques suffisamment homogènes entre elles pour définir un "faciès de production "pouvant par ailleurs se rapporter à une ou plusieurs entités culturelles (Bodu et Valentin, 1997 ; Bodu et al., 1997; Bodu [dir.], 1998 ; Fagnart, 1997a et b; Hantaï, 1994 ; Valentin, 1995).

\section{Des affinités dans les choix techniques}

Même si les traditions à Federmesser et belloisiennes partagent toutes deux l'usage du percuteur de pierre tendre pour le débitage laminaire, elles se différencient profondément au travers de comportements techniques et économiques radicalement distincts. Que ce soit dans la sélection rigoureuse des matières premières, dans les procédés élaborés de mise en forme ou dans les méthodes d'exploitation laminaire, les gisements belloisiens présentent des convergences techniques indiscutables (Valentin, 1995). En intégrant les données du Closeau aux gisements déjà connus et récemment documentés ${ }^{10}$, nous montrerons combien les données du Closeau rejoignent pour partie les observations déjà effectuées tout en nous attachant à retranscrire ce qui fait l'originalité de ce gisement des bords de Seine.

Une caractéristique partagée par l'ensemble des industries belloisiennes réside dans l'exploitation d'au moins une variété de silex local, remarquable par sa qualité et ses dimensions importantes (Hantaï, 1994 ; Valentin et al., 1997). La présence de tels matériaux à proximité des gisements expliquerait en partie l'implantation des paléolithiques venus satisfaire leurs besoins en grandes lames (Valentin, 1995). Toutefois, cette explication n'est pas décisive dans tous les cas comme en témoignent les gisements de Rilly-Sainte-Cyr (Aube) et Acquigny-La-Noë (Eure) où le silex d'origine alluviale est de bonne qualité sans pour autant atteindre les caractéristiques exceptionnelles des volumes de Belloy-surSomme (Somme) ou Donnemarie-Dontilly (Seine-etMarne) par exemple (Valentin, 1995). Au Closeau, l'exploitation largement dominante d'une variété de silex local est confirmée par la domination des silex secondaires du Campanien. Ces matériaux présents en quantité à l'aplomb du site présentent une aptitude correcte à la taille, sans être toutefois d'une qualité exceptionnelle. Par ailleurs, les tailleurs du Closeau ont préféré les volumes de petites à moyennes dimensions (10 à $25 \mathrm{~cm}$ ), délaissant les plus gros pourtant présents sur les mêmes lieux d'acquisition. Même si tous ces gisements présentent à proximité immédiate une source de matière première de qualité acceptable, il n'en reste pas moins qu'une diversité peut être mise en évidence au niveau des dimensions et de la qualité des volumes exploités. Lorsque ces deux critères sont réunis dans un même assemblage (Belloy-sur-Somme, Flixecourt, Donnemarie-Dontilly), l'évocation d'une spécialisation dans les activités de taille est introduite pour expliquer ces comportements (Valentin, 1995). Toutefois, cette spécialisation particulièrement claire dans certains cas l'est moins sur d'autres gisements comme le Closeau par exemple, où l'investissement n'est pas particulièrement porté sur la qualité ou les dimensions des volumes exploités. Nous garderons donc l'idée qu'il peut exister une "spécialisation multiple" (Valentin, 1995, p. 694), différente selon les gisements, pourtant tous rattachés à la même tradition technique. Cette optique sera ultérieurement réexaminée à la lueur notamment de l'analyse des pointes à dos, inégalement représentées suivant les gisements. C'est plus particulièrement dans la conduite du débjtage que nous retrouvons les liens de parenté les plus évidents entre les gisements belloisiens comme la distinction la plus radicale avec les groupes a Federmesser. La méthode employée est en relation avec des objectifs, techniquement contraignants de ce point de vue. L'obtention de lames au profil rectiligne nécessite en effet l'emploi de méthodes adaptées, nécessitant un bon degré de savoir-faire. Suivant les cas et surtout la nature des matériaux utilisés, les phases de mise en forme peuvent être plus ou moins conséquentes. Bien qu'il existe toujours certaines chaînes opératoires simplifiées, notamment pour l'exploitation de petits volumes dont la morphologie initiale permet une entame rapide du débitage laminaire, les industries belloisiennes se caractérisent fréquemment par l'intensité de mise en forme des volumes incluant une "assez longue séquence d'initialisation" (Valentin, 1995, p. 696). Au Closeau, cette option peut être mise en évidence à partir des produits d'aménagement des nucléus permettant de distinguer, dans certains cas, une séquence de dégrọssissage suivie d'une séquence de cadrage. Toutefois, les volumes traités au Closeau présentent le plus souvent un compromis idéal entre la morphologie régulière des nodules et la configuration recherchée pour l'obtention des supports utilitaires. C'est pourquoi il est relativement courant que l'aménagement des nucléus soit simplifié, modalité déjà mise en évidence sur l'ensemble des sites belloisiens.

Il en est de même lors des séquences de plein débitage qui, au Closeau, peuvent fréquemment être conduites à partir d'un plan de frappe préférentiel et selon une dynamique relativement frontale à partir d'une surface laminaire à la carène peu prononcée et au cintre ouvert. Toutefois, sur de plus gros volumes, le débitage est conduit depuis deux plans de frappe opposés installés dès le début de l'exploitation et progresse selon un 

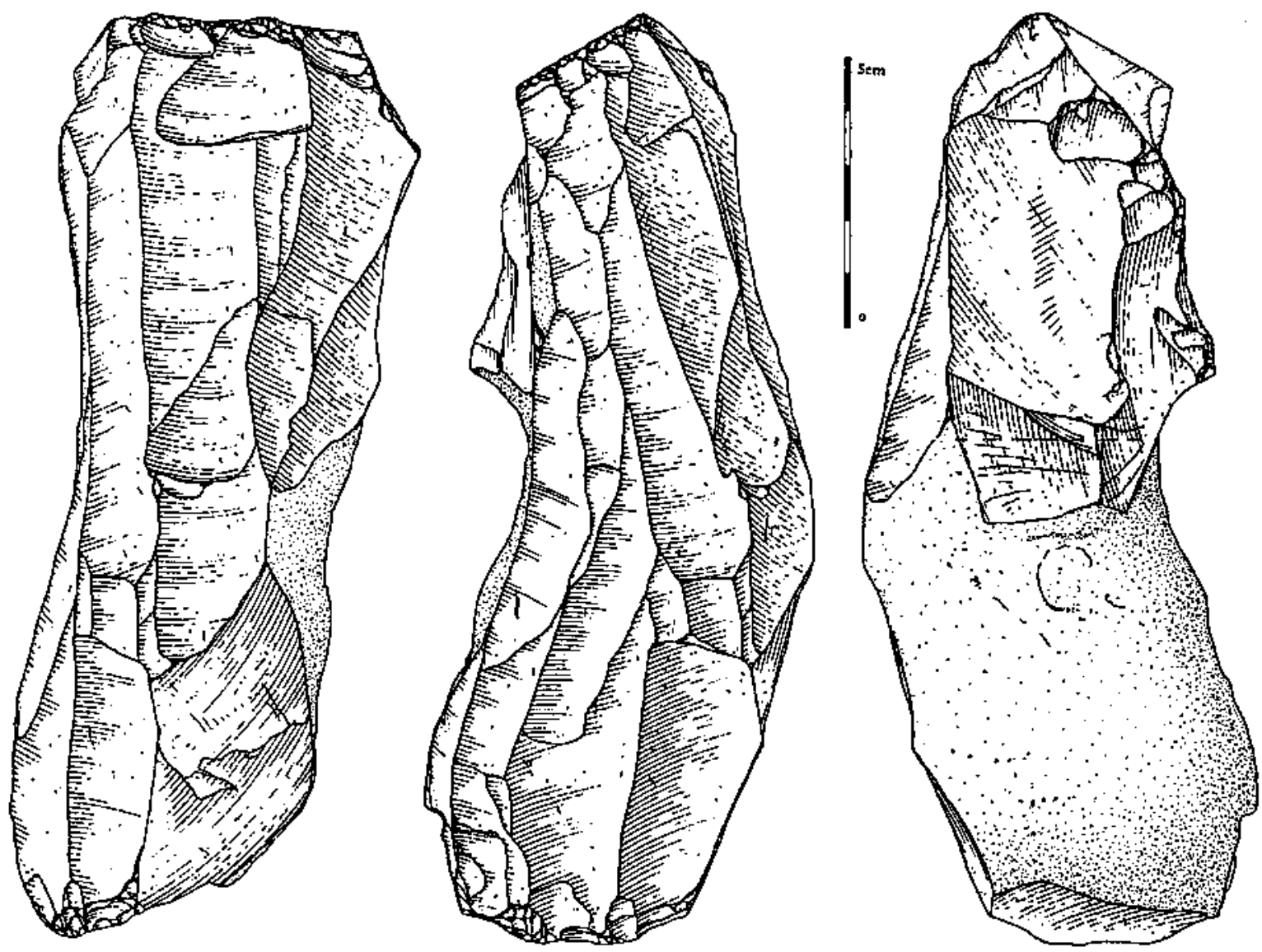

Fig. 7 - Le Closeau, secteur Sud R.N. 13 : Nucléus à lames : granđ nucléus à lames à deux plans de frappe opposés (dessin : P. Alix).

rythme fréquemment semi-tournant, parfois sécant, mettant en jeu plusieurs surfaces de débitage (fig. 7). La préparation au détachement des lames est effectuée par abrasion systématique en direction de la surface laminaire, parfois renforcée par un véritable doucissage. Le percuteur de pierre tendre vient accrocher le bord du plan de frappe selon une gestuelle tangentielle, se rapprochant de celle usitée avec un percuteur tendre organique. Le soin apporté à la conduite du débitage laminaire précise d'autant plus les affinités du Sud R.N. 13 du Closeau avec d'autres ensembles belloisiens qu'il constitue l'un des fondements caractérisant cette "tradition" (Bodu et al., 1997; Fagnart, 1997b ; Valentin, 1995). La disparité constatée au Closeau (entre des exploitations parfois unipolaires ou bipolaires, frontales ou semi-tournantes, voire sécantes) tient peut-être dans la moindre dimension des volumes exploités, ne nécessitant pas toujours la mise en œuvre de tels procédés. Le gisement du Closeau présente, au niveau technique, des points de convergence intéressants avec le faciès des "industries à pièces mâchurées". Les dimensions moyennes des volumes exploités confèrent un caractère moins gigantesque à cette industrie au regard des autres industries belloisiennes de la Somme ou de Donnemarie-Dontilly. Toutefois, outre la diversité dans la qualité et les dimensions des matières premières, une parenté technique unit l'ensemble de ces gisements, préférentiellement orienté vers une production laminaire standardisée. À ce propos, B. Valentin précisait que "la longueur fait probablement partie des exigences mais elle ne peut être considérée comme prioritaire dans la hiérarchie des options: les lames les plus longues sont irrégulières et très robustes et elles ont été généralement considérées comme des déchets "(Valentin, 1995, p. 699). Les produits laminaires du Closeau satisfont pleinement cette assertion et relativisent l'emploi de la terminologie anglo-saxonne ("Long Blade Techno$\log y$ ").

Cependant, même si la longueur des lames n'est que relative, l'industrie du Closeau présente certaines particularités, que ce soit dans la simplification de certains débitages laminaires ou dans le changement d'orientation du đébitage avant l'abandon des nucléus, perceptible dans le nombre de nucléus finissant en nucléus à éclats. Cette diversité, difficilement compréhensible par une simple analyse technique, peut trouver des moyens d'expression dans la vocation économique des activités de taille du silex, toujours dominante.

\section{Des affinités dans les choix économiques}

Classiquement, les séries belloisiennes ont été essentiellement définies par leur particularité économique : on parle d'ateliers de débitage du silex, à proximité des 
sources de matières premières, se traduisant par une rareté en produits retouchés contrastant avec l'abondance des produits débités (Fagnart, 1997a). Une valeur forte a par ailleurs été conférée à des "outils a posteriori ", les lames mâchurées servant selon les dernières analyses tracéologiques et expérimentales à l'aménagement et l'entretien des percuteurs de pierre tendre (Fagnart et Plisson, 1997). Ces pièces ne sont cependant pas suffisantes pour une caractérisation fine de ce complexe. En témoignent leur relative inconstance selon les sites considérés et leur présence sporadique dans d'autres industries non contemporaines. Au Closeau, sur plus de 18000 artefacts lithiques, on ne compte que 17 pièces mâchurées. Deux autres facteurs peuvent maintenant être développés dans la caractérisation de ces séries : il s'agit de la rareté en outils retouchés et en produits laminaires bruts de première intention.

Selon les industries belloisiennes, le pourcentage d'outils retouchés volontairement varie entre 0,3 et $2,5 \%$ (Valentin, 1995). Au Closeau, les données du locus P font état d'un pourcentage comparable puisque les outils constituent $1,66 \%$ de l'assemblage. À ces faibles taux s'ajoute une relative monotonie des types d'outils rencontrés plaiđant pour une restriction des champs d'activités (Valentin, 1995). En règle générale, les grattoirs et les denticulés constituent l'essentiel des outils du fond commun, contrastant avec la rareté des becs et perçoirs si fréquents dans les assemblages magdaléniens. Par ailleurs, une des divergences majeures avec d'autres industries tardiglaciaires réside dans la rareté des armatures (Bodu et al., 1997 ; Fagnart, 1997a et b; Hantaï, 1994; Valentin, 1995). Ces témoignages concordent avec les données relevées au Closeau, à l'exception notable des armatures qui constituent une classe d'outils dominante. Nous reviendrons ultérieurement sur ce point et sur la valeur des pièces à dos dans l'identification des occupants du site.

Ả la rareté des outils retouchés volontairement s'ajoute une carence en produits de première intention. Cette carence est telle qu'au Closeau, et il en est de même sur d'autres gisements, elle est perceptible uniquement à travers l'observation de l'ensemble de la production". Nous avons déjà évoqué que sur le locus $P$, seules 22 lames de plein débitage nous étajent parvenues entières. P. Bodu a réalisé des observations similaires sur l'ensemble des locus du Sud R.N. 13 (Bodu [dir.], 1998). Même si nos tentatives n'ont pu être répétées, il semble particulièrement difficile de raccorder entre eux les produits laminaires. Enfin, confronté à la quantité de nucléus à projet laminaire, le nombre de lames entières de plein débitage paraît dérisoire. C'est pourquoi, nous pensons à des prélèvements systématiques et d'ampleur importante des produits laminaires de première intention.

B. Valentin précise pour d'autres industries belloisiennes (notamment Donnemarie-Dontilly et Muidessur-Loire) que, "quand on a pu le certifier, les prélèvements semblent d'assez grande ampleur, sans commune mesure avec ce que l'on connaît dans d'autres faciès tardiglaciaires régionaux" (Valentin, 1995, p. 690). J.-P. Fagnart aboutit à des conclusions similaires définissant le niveau à pièces mâchurées de Belloy-sur-Somme comme "une succession de postes de taille spécialisés dans la production de lames, dont une partie est emportée à l'extérieur du gisement pour une utilisation différée " (Fagnart, 1997a, p. 226).

Comme nous venons de le souligner, la série lithique du gisement du Closeau, secteur Sud R.N. 13, partage différentes affinités avec un faciès d'activités spécialisées défini sous le terme neutre de Belloisien. Ces rapprochements peuvent être effectués en vertu d'une "ambiance technique" comparable répondant pour partie à des choix économiques spécifiques. Il reste maintenant à poser la question de 1 '“identité culturelle" des acteurs de cette tradition. Certains auteurs ont déjà indiqué la difficulté de cette entreprise, rendue délicate par la pauvreté de ces industries en pièces retouchées (Bodu et al., 1997 ; Fagnart, 1997b; Valentin 1995, 1999). La domination d'outils aux caractères ubiquistes prononcés ne facilite pas cette démarche. C'est peut-être de ce point de vue que le gisement đu Closeau signe toute son originalité. Il présente la particularité d'offrir un nombre relativement élevé, pour les industries belloisiennes, d'outils reconnus comme de véritables marqueurs culturels : les pointes à dos. L'échantillon du locus $P$ étant restreint et fragmenté, nous nous sommes permis d'élargir notre corpus d'étude à tous les locus du Sud R.N. 13. Cet élargissement nous permet maintenant de préciser la particularité de ces pointes et de les replacer dans le panorama actuel de la fin du Tardiglaciaire.

\section{APPORT DES POINTES À DOS DANS LA RECHERCHE SUR L" IDENTITÉ CULTURELLE" DES OCCUPANTS DU CLOSEAU, SUD R.N. 13}

Sur l'ensemble đu matériel attribué aux locus synchrones du locus $\mathrm{P}, 50$ pointes à dos ${ }^{12}$ ont été reconnues. Il s'agit, sauf rares exceptions, d'exemplaires très fragmentés. Toutefois, il apparaît selon plusieurs caractères que ces pièces forment un ensemble suffisamment homogène pour mériter une présentation commune. Au niveau typologique d'abord, plus de $95 \%$ de ces pièces appartiennent à la catégorie des "pointes à dos rectiligne". Contrairement aux industries aziliennes du Closeau, les pointes à dos courbe revêtent jci un caractère plus marginal. Au niveau morphologique et stylistique enfin, ces exemplaires présentent des attributs diagnostiques relativement stables (délinéation du dos, épaisseur du dos, type de support sélectionné) permettant a priori de rattacher l'ensemble de ce corpus à une même tradition technique (fig. 8). Pour l'ensemble des locus, le groupe des armatures à dos peut être subdivisé de la façon suivante (tabl. 4). Comme nous l'avions déjà souligné lors de l'étude du locus $P$, nous constatons une nette domination des pointes à dos rectiligne sur les pointes à dos courbe. Ces pointes, pour l'essentiel fragmentées, sont aménagées sur de petites lames de plein débitage toujours très régulières. Cette standardisation des supports trouve 


\begin{tabular}{|l|c|c|c|c|c|c|}
\cline { 2 - 7 } & $\begin{array}{c}\text { Pointes à dos } \\
\text { rectiligne épais } \\
(>4 \mathrm{~mm})\end{array}$ & $\begin{array}{c}\text { Pointes à dos } \\
\text { rectiligne mince } \\
(<4 \mathrm{~mm})\end{array}$ & $\begin{array}{c}\text { Pointes à dos } \\
\text { rectiligne et } \\
\text { base tronquée }\end{array}$ & $\begin{array}{c}\text { Pointes à } \\
\text { dos courbe }\end{array}$ & $\begin{array}{c}\text { Lamelles } \\
\text { à dos }\end{array}$ & Total \\
\hline Entières & 2 & 1 & 2 & 1 & 0 & 6 \\
\hline Fragments basaux & 1 & 3 & 1 & 0 & 0 & 5 \\
\hline Fragments mésiaux & 7 & 9 & & 1 & 4 & 21 \\
\hline Fragments apicaux & 8 & 8 & & 2 & 0 & 18 \\
\hline Total & 18 & 21 & 3 & 4 & 4 & 50 \\
\hline
\end{tabular}

Tabl. 4 - Le Closeau, secteur Sud R.N. 13 : présentation des pointes à dos de tous les locus.
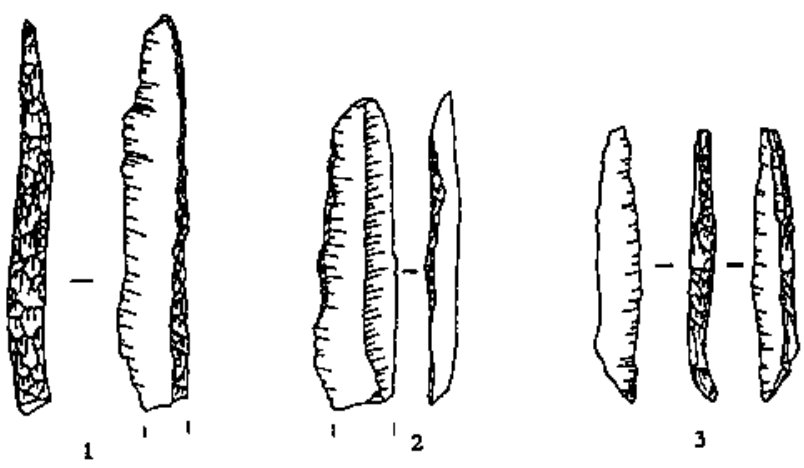

3
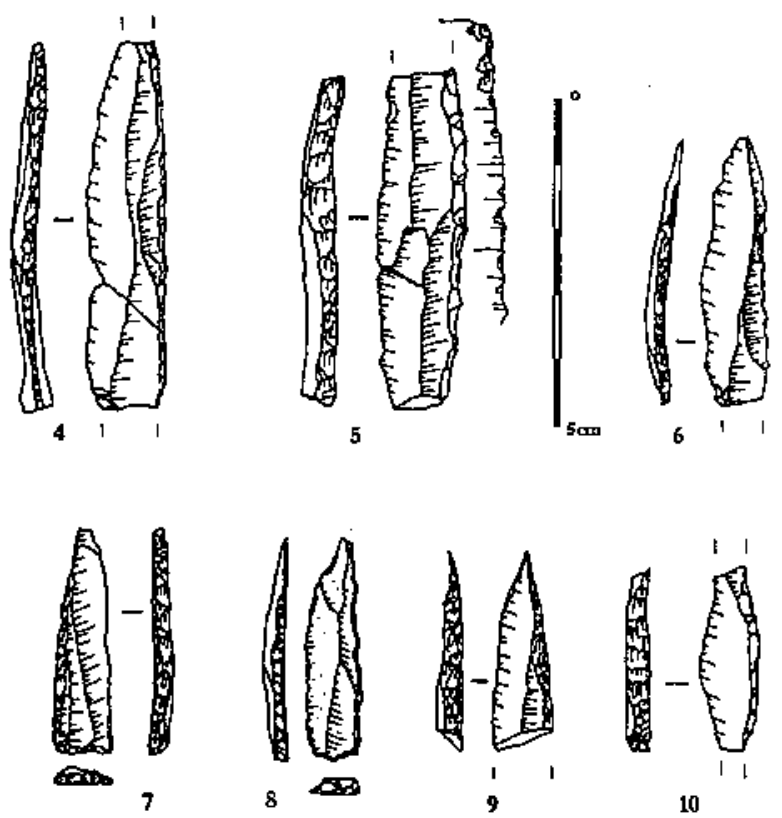

Fig. 8 - Le Closeau, secteur sud R.N. 13 : Pointes à dos : 1-2, 4-5, 9 -10 : pointes à dos rectiligne ; 3 ; pointe des Blanchères ; $7-8 ;$ pointes de Malaurie (dessins: P. Alix et F, Kildéa).

apparemment son corrélât dans le fait que, dans la majorité des cas, la retouche ne semble pas modifier de façon significative la délinéation du bord aménagé, notamment dans l'exemple des pointes à dos rectiligne mince. Au niveau de la composition des types de pointes rencontrées, nous signalons la présence d'au moins trois pointes de Malaurie (fig. $8, \mathrm{n}^{\text {os }} 7-8$ ) ainsi qu'une petite pointe à dos rectiligne, plus étroite que les autres pointes à dos et présentant à sa base un léger aménagement par retouche inverse (fig. $8, n^{\circ} 3$ ).
Comme le constatait P. Bodu (Bodu [dir.], 1998), cette pièce présente de remarquables affinités avec le type créé par J.-G. Rozoy et nommé pointe des Blanchères (Rozoy, 1978). Sur le site éponyme, ces pointes se rencontrent en association avec des pointes de Malaurie (Valentin, 1995).

Dans le panorama actuel du Tardiglaciaire, les pointes à dos rectiligne sont plus particulièrement associées aux derniers épisodes de cette période. Lors du développement des groupes à Federmesser, ces pointes sont peu représentées et les assemblages dominés largement par les pointes à dos courbe donnant leur nom à ce technocomplexe (Bodu et Valentin, 1997 ; Fagnart, 1997a et b ; Thévenin, 1997 ; Valentin, 1995). Cette particularité typologique semble dès lors aller dans le même sens que nos observations techniques s'attachant à montrer les profondes différences entre l'assemblage du Closeau Sud R.N. 13 et les procédés techniques utilisés par les groupes à Federmesser ou aziliens, que ce soit au Closeau ou sur d'autres gisements récemment étudiés. Ce n'est que lors du dernier épisode froid du Dryas récent qu'apparaissent, dans le nord du Bassin parisien, plusieurs "faciès" à pointes de Malaurie (Fagnart, 1997a). Cette évolution dans la morphologie des pointes à dos (đu dos courbe vers le dos rectiligne) a été observée lors du Dryas récent dans d'autres régions puisque l'on observe un processus similaire sur le site de la Fru (Pion, 1990) en Savoie ou dans le sud-ouest de la France (Célérier, 1993 et 1998 ; Le Tensorer, 1981). Ces observations étendues à diverses régions ont amené différents auteurs (Bodu et al, 1997 ; Hantaï, 1994; Valentin, 1995) à mettre en relation les industries belloisiennes avec un faciès à pointes de Malaurie reconnu dans le sud-ouest de la France, le Laborien (Le Tensorer, 1981). Pour l'heure, cette hypothèse n'était pas particulièrement bien étayée par les données archéologiques, compte tenu du faible nombre de pointes à dos recueillies dans ces contextes. À Donnemarie-Dontilly, deux armatures seulement ont été découvertes (lamelles à dos étroites) et à Muides-surLoire, les témoignages sont en nombre équivalent puisque deux pointes à dos rectiligne, dont une porte une base tronquée, sont signalées par A. Hantaï (Hantaï, 1994). Toutefois la présence d'une pointe de Malaurie avait permis de proposer une première filiation entre cette industrie rapportée au Belloisien et un faciès plus "méridional" reconnu dans le sud-ouest de la France à la Borie-del-Rey, site éponyme du Laborien. Les possibilités de comparaison des gisements spécialisés du Belloisien se trouvaient ainsi élargies à d'autres 
domaines que l'Ahrensbourgien, première hypothèse de filiation proposée par les auteurs anglais (Barton, 1989) et J.-P. Fagnart (Fagnart, 1997a et b) pour le bassin de la Somme.

Les données recueillies au Closeau corroborent les propositions effectuées par P. Bodu, A. Hantaï et B. Valentin. Dans un premier temps, l'hypothèse de R.N. Barton (Barton, 1989) pour les gisements à pièces mâchurées du sud-est de 1'Angleterre et de J.-P. Fagnart (1997a et b) pour les sites belloisiens du bassin de la Somme d'une affinité avec le complexe Ahrensbourgien ne trouve aucun élément probant de validation dans la composition des pointes à dos du Closeau. Aucune armature d'affinités ahrensbourgiennes n'a pu être observée. En revanche, la domination presque exclusive des pointes à dos rectiligne avec l'association certes minoritaire de pointes de Malaurie évoquent le Laborien décrit par J.-M. Le Tensorer à partir de la couche 5 du site de La Borie-del-Rey (Le Tensorer, 1981). Dans cette industrie, les grattoirs sont peu nombreux mais toujours courts et les armatures nombreuses indiquent la prédominance des pointes de Malaurie. Enfin, J.-M. Le Tensorer signale la présence de couteaux à dos, en petit nombre mais selon lui très caractéristiques. Des pièces similaires se retrouvent, également en petite quantité, dans l'industrie du Closeau que nous venons d'étudier. Enfin, cette occupation laborienne de la Borie-del-Rey a été datée par ${ }^{14} \mathrm{C}$ de $10350 \pm 340$ B.P., la plaçant au cours du Dryas récent. Un autre gisement stratifié du sud-ouest de la France apporte maintenant de précieux renseignements; il s'agit du site de Pont d'Ambon en Dordogne septentrionale (Célérier, 1993 et 1998). C'est notamment la couche 2 datée par ${ }^{14} \mathrm{C}$ de $9640 \pm 120$ B.P. (cal. 9854 8416 B.C.) qui nous intéresse ici puisqu'elle a été considérée comme un Azilien terminal d'affinités laboriennes (Célérier, 1998), compte tenu de la présence de pointes de Malaurie et de l'émergence des rectangles à dos. Il faut toutefois constater que d'après les datations absolues, l'occupation laborienne de Pont d'Ambon est plus tardive de près d'un millénaire de celle du gisement éponyme. Il convient à cet effet de considérer ces comparaisons avec une grande prudence et de garder à l'esprit que certaines dates des gisements du sud-ouest français sont éventuellement sujettes à caution. Nous disposons pour la couche 2 de Pont d'Ambon de données technologiques indiquant un débitage laminaire de bonne qualité destiné ả fournir des supports rectilignes à l'aide de nucléus à deux plans de frappe opposés. Il est intéressant de constater que, comme dans le Nord de la France, cette industrie est en totale rupture avec les couches sous-jacentes, présentant un débitage peu organisé conduit à la percussion directe dure. Cette analogie renvoie selon nous à ce qu'il est possible d'observer en Ile-de-France ou dans le bassin de la Somme à la fin de la séquence des groupes à Federmesser.

Comme nous venons de l'observer, l'assemblage des pointes à dos rectiligne du secteur Sud R.N. 13 du Closeau nous renvoie vraisemblablement à un ensemble de séries datées du Dryas récent ou de sa transition avec le Préboréal. Ces ensembles sont toujours en rupture avec les industries les précédant, se distinguant par un faible investissement technique placé dans les activités de taille du silex. Nous sommes conscient des limites de notre échantillon qui demeure tout aussi faible quantitativement que qualitativement, étant donné son intense fragmentation. Toutefois, si la proposition de rattacher cet ensemble à la tradition des groupes belloisiens se confirme par les données techniques, elle l'est tout autant par le caractère diagnostique de ces armatures, qui se différencient nettement de la tradition des groupes à Federmesser. Nous constatons également qu'au niveau chronologique, les ensembles belloisiens paraissent synchrones avec le complexe Laborien, tous deux situés à la charnière entre le Dryas récent et le Préboréal.

Nous percevons peut-être mieux maintenant la question đe l'identité culturelle des occupants des gisements belloisiens. Aux premières propositions établies par quelques auteurs sur la possible filiation de ces ensembles avec les industries laboriennes, viennent s'ajouter les données du Closeau et leur cortège de pointes à dos. Il n'en reste pas moins que de nombreuses réserves peuvent être émises sur ces filiations, compte tenu de la distance importante séparant les gisements belloisiens les plus méridionaux et les ensembles laboriens du sud-ouest de la France. Les prochaines recherches en Ile-de-France doivent maintenant se consacrer, pour ce faciès particulier, à l'identification des sites d'habitat s'en rapprochant. C'est ainsi qu'en reprenant l'étude d'une série connue depuis longtemps, B. Valentin a pu proposer d'intéressants rapprochements entre le site d'habitat des Blanchères dans les Yvelines et le faciès belloisien (Valentin, 1995). Le gisement des Blanchères mais aussi celui de la Muette 1 à Vieux-Moulin (Oise) pourraient correspondre aux habitats des gisements spécialisés dans les activités de taille du silex du Belloisien. Notons par ailleurs que pour la première fois sur un ensemble belloisien, il a été possible au Closeau de mettre en évidence une association reconnue aux Blanchères entre pointes de Malaurie et pointes des Blanchères. Certes remarquable, cette association garde pour l'heure un caractère particulier puisqu'elle n'est basée que sur la présence d'une seule pointe des Blanchères.

\section{CONCLUSION}

L'étude d'une série lithique du secteur Sud R.N. 13 du Closeau a permis de retrouver d'intéressantes affinités tant techniques qu'économiques avec un ensemble de gisements particulièrement bien représentés dans le sud-est de l'Angleterre et dans le bassin de la Somme, provisoirement désignés sous le terme de Belloisien. Au regard des datations absolues replaçant assez systématiquement ces industries entre le Dryas récent et le Préboréal, nous sommes désormais en mesure de nous poser la question de l'identité culturelle des tenants de cette "tradition". Compte tenu de la faible proportion d'outils retouchés au sein de ces industries, cette recherche se trouve actuellement confrontée aux 
limites des études technologiques en terme de marqueur culturel. Toutefois, la mise en ouvre d'approches techno-économiques, associant les modalités du débitage et leurs objectifs, permet déjà d'aboutir à d'intéressantes constatations. Grâce à ces acquis analytiques, il a notamment été possible d'établir des liens de convergence entre les industries belloisiennes du bassin de la Somme (Belloy, Hangest, Flixecourt), celles du Bassin parisien (Donnemarie-Dontilly, Le Closeau) et d'autres dans un contexte plus méridional (Muides-sur-Loire). On peut aisément imaginer ce qui aurait été déduit de ces industries lorsque seule était en vigueur la typologie de l'outillage et se rendre compte des profondes avancées que nous a permis l'usage non exclusif de la technologie lithique. En revanche, et nous le percevons bien lorsqu'il s'agit de rechercher

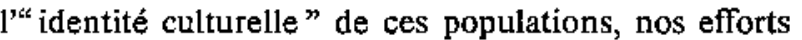
deviennent plus difficiles encore lorsqu'il s'agit de dépasser ces rapprochements inter-sites et d'accéder à leur compréhension. Que signifie par exemple la "rupture" si souvent évoquée entre les groupes aziliens de la fin de l'Alleröd et les traditions techniques reconnues lors du Dryas récent et du Préboréal ? Pourquoi, en un laps de temps relativement court, passe-ton d'inđustries peu standardisées à un débitage de grandes lames rectilignes? Et c'est assurément pour aborder de telles problématiques que la technologie lithique devra être replacée au sein d'approches pluridisciplinaires plus globales intégrant l'Homme et son environnement. Il faudra pour cela découvrir des sites favorables à de telles entreprises, ce qui n'était pas le cas pour le secteur Sud R.N. 13 du Closeau où seule l'étude des silex taillés était rendue possible par les procédés taphonomiques ayant abouti à la destruction de toutes les autres catégories de vestiges.

\section{Remerciements}

Je remercie très sincèrement Pierre Bodu pour la confiance qu'il m'a témoignée en me permettant de travailler sur le Closeau et pour son aide toujours très efficace, Jacques Pelegrin pour sa relecture du premier manuscrit et Jean-Pierre Fagnart d'avoir accepté d'être rapporteur de cet article. Mes remerciements s'adressent également à Magen O'Farrell pour son aide lors de la traduction du résumé en anglais et à Caroline Renard pour ses corrections et son aide dans l'illustration de cet article.

\section{NOTES}

(1) Depuis, de nouvelles dates sont venues confirmer la place du niveau inférieur au début de l'Alleröd avec pour le locus 46 les résultats suivants : $12350 \pm 60$ B.P. (cal. $12836-12191$ B.C.) et $12360 \pm 60$ B.P. (cal. $12850-12203$ B.C.) (Bodu, comm. personnelle).

(2) Le terme "Belloisien" désignant un faciès techno-économique du Paléolithique final est noté en italique pour bien le dèmarquer dés grands techno-complexes du Paléolithique supśrieur (Solutréen, Magdalénien...) qui ne procèdent pas du même niveau de définition. (3) Les résultats présentés sont en grande partie empruntès à un travail universitaire de D.E.A. (Teyssandier, 1999).

(4) Ont été considérés comme des lames de plein débitage, tous les produits laminaires ne portant pas de traces de cortex ou de négatifs d'enlèvements transversaux au sens du détachement.

(5) Grâce à l'aide de P. Bodù et J. Pelegrin que nous remercions. (6) $73 \%$ des talons de james de plein débitage sont lisses dont $72 \%$ lisses abrasés, $17 \%$ punctiformes abrasés et $11 \%$ simplement lisses. (7) L'ensemble du Sud R.N. 13 n'a pas livré de charbons de bois ou de restes osseux. Par ailleurs, la stratigraphie ne permet pas de replacer l'horizon archéologique au sein d'une chronologie relative.

(8) Site occupé uniquement, pour les périodes palèolithiques, durant la seconde partie du Tardiglaciaire, approximativement entre 12000 et 10000 B.P.

(9) Ces industries sont également dénommées "inđustries à pièces mâchurées " (Bodu, Hantaï et Valentin, 1997 ; Fagnart, 1997a et b) ou "Long Blade Technology" selon la dénomination des chercheurs anglais (Barton, 1989).

(10) Notamment dans la Somme avec les sites de Belloy, Conty, Flixecourt et Hangest (Fagnart 1997a), dans le bassin de la Tamise avec les sites de Sproughton et Avington VI (Barton, 1989 ; Dumont, 1997), en Ile-de-France avec Donnemarie-Dontilly (Valentin, 1995) et plus au sud dans le Loir-et-Cher sur le site de Muides-sur-Loire (Hantaï, 1994).

(11) Il n'est pas obligatoire, bien que cela permettrait de quantifier plus finement ce phénomène, de procéder à des remontages pour l'attester.

(12) Nous remercions ici sincèrement $M$. Jean-Betnard Roy, Conservateur du Musée de Nemours, de nous avoir permis et facilité l'accès au matériel des autres locus du Closeau.

\section{RÉFÉRENCES BIBLIOGRAPHIQUES}

AUDOUZE F. et al. (1988) - Taille du silex et finalité du débitage dans le Magdalénien du Bassin parisien, in : M. Otte (éd.) : De la Loire d̀ l'Oder: les civilisations du Paléolithique final dans le nord-ouest européen. Actes du colloque international de Liège (déc. 1985), E.R.A.U.L., 25, B.A.R. International Series, 444, p. 55-84.

BARTON R.N.E. (1989) - Long Blade Technology in Southern Britain, in: C. Bonsall (éd.), The Mesolithic in Europe. Edinburgh, 1985 , p. 264-271, 5 fig.

BARTON R.N.E. et ROBERTS A.J. (1997) - Systèmes économiques et modalités techniques dans l'ouest de la Grande-Bretagne au cours du Tardiglaciaire, in : J.-P. Fagnart et A. Thévenin (dir.), Le Tardiglaciaire en Europe du Nord-Ouest, Áctes du $119^{\circ}$ congrès national des Sociétés Historiques et Scientifiques, Amiens, 1994, Paris, C.T.H.S., p. 507-516, 6 fig.

BODU P. (dir.) (1998) - "Le Closeau", deux années de fouille sur un gisement azilien et belloisten en bord de Seine. Document Final de Synthèse de sauvetage urgent, A.F.A.N., S.R.A d'Ile-de-France, ex. multigraph., 3 vol.

BODU P. et VALENTIN B. (1992) - L'industrie à pièces mâchurées de Donnemarie-Dontilly (Seine-et-Marne, France) : un faciès tardiglaciaire inédit dans le Bassin parisien, Préhistoire européenne, 1, p. 15-23.

BODU P. et VALENTIN B. (1993) - Nouveaux résultats sur le site tardiglaciaire a lames máchurées de Donnemarie-Dontilly (Seine-et-Marne), Préhistoire européenne, 4, p. 85-92.

BODU P. et VALENTIN B. (1997) - Groupes à Federmesser ou aziliens dans le Sud et l'Ouest du Bassin parisien. Propositions pour un nouveau modèle d'évolution, Bulletin de la Société Préhistorique Française, t. 94, p. 341-347, 2 fig.

BODU P., JULIEN M. et VALENTIN B. (1994) - Projet collectif de recherche sur les habitats tardiglaciajres du Bassin parisien: bilan des travaux récents, Bulletin de la Société Préhistorique Française, t. 91, p. 182-184.

BODU P., HANTAÏ A. et VALENTIN B. (1997) - La Long Blade Technology au sud du Bassin parisien : découvertes récentes, in : J.-P. Fagnart et A. Thévenin (dir.), Le Tardiglaciatre en Europe du Nord-Ouest, Actes du $119^{\circ}$ congrès national des Sociétés Historiques et Scientifiques, Amiens, 1994, Paris, C.T.H.S., p. 211-222, $5 \mathrm{fig}$. 
CÉLÉRIER G. (dir.) (1993) - L'abri sous roche de Pont d'Ambon à Bourdeilles (Dordogne). I: Technologie de l'outillage lithique taillé ; II : Inventaire et typométrie des pointes aziliennes; III : Aralyse technologique du matériel de la couche 3B; IV : Le matériel lithique non taillé, Gallia-Préhistoire, 35, C.N.R.S., p. $1-137$.

CÉLERIER G. (1998) - L'abri sous roche de Pont d'Ambon a Bourdeilles (Dordogne, France). Perspective synthétique, Paléo, 10, p. $233-264,22$ fig.

DUMONT S. (1997) - Nouvelles recherches sur ta transition Tardiglaciaire-Préboréal dans le Sud et l'Est de l'Angleterre, in : J.-P. Fagnart et A. Thévenin (dit), Le Tardiglaciaire en Europe du Nord-Ouest, Actes du $119^{\circ}$ congrès national des Sociétès Historiques et Scientifiques, Amiens, 1994, Paris, C.T.H.S., p. 517-527, 7 fig.

FAGNART J.-P. (1997 a) - La fin des temps glaciaires dans le Nord de la France. Approches archéologique et environnementale des occupations humaines du Tardiglaciaire, Mémoires de la Société Préhistorique Française, t. 24, 270 p., 182 fig.

FAGNART J.-P. (1997 b) - Palèohistoire du bassin de la Somme à la fin des temps glaciaires, in: J.-P. Fagnart et A. Thévenin (dir.), Le Tardiglaciaire en Europe du Nord-Ouest, Actes du $119^{E}$ congrès national des Sociétés Historiques et Scientifiques, Amiens, 1994, Paris, C.T.H.S., p. 55-77, 9 fig.

FAGNART J,-P. et PLISSON H. (1997) - Fonction des pièces mâchurées du Pạléolithique final du bassin de la Somme. Caractères tracéologiques et données contextuelles, in: J.-P. Fagnart et A. Thévenin (dir.), Le Tardiglaciaire en Europe du Nord-Ouest, Actes du $119^{\circ}$ congrés rational des Sociétès Historiques et Scientifiques, Amiens, 1994, Paris, C.T.H.S., p. 95-106, 4 fig, 5 photos.

HANTAÏ A. (1994) - La Long Blade Technology jusqué sur les bords de la Loire: approche techno-économique de deux séries lithiques recueillies à Muides-sur-Loire (Lotr-et-Cher). Mémoire de D.E.A., Université de Paris l, ex. muItigraph., 129 p.

LE TENSORER J.-M. (1981) - Le Paléolithique de l'Agenais, Paris, C.N.R.S., (Cahiers du Quaternaire, 3), 526 p.

PELEGRIN J. (1995) - Le Châtelperronien de Roc-de-Combe (Lot) et de la Cóte (Dordogne), Paris, C.N.R.S., (Cahiers du Quaternaire, 20), $297 \mathrm{p}$.

PELEGRIN J. (sous presse) - Les techniques de đébitage laminaire au Tardiglaciaire : critères de diagnose et quelques réflexions, in: B. Valentin., P. Bodu et M. Christensen (éd.), Actes de la table-ronde de Nemours maj 1997.
PERLES C. (1987) - Les industries lithiques tailiées de Franchti. 1: Présentation générale et industries paléolithiques, Bloomingtoni Indianapolis, Indiana University Press, (Excavations at Franchti Cave, Greece), fasc. 3, 355 p.

PION G. (dir.) (1990) - L'abri de la Fru à Saint-Christophe (Savoie), Gallia-Préhistoire, 32 , p. 65-123.

ROZOY J.-G. (1978) - Les derniers chasseurs. L'Épipaléolithique en France et en Belgique. Essai de synthèse, Bulletin de la Socièté Archeologique Champenoise, 3 vol., 1256 p.

TEYSSANDIER N. (1999) - Le Closeau, secteur Sud R.N. 13: une occupation de l'extrême fin du Tardiglacialre en He-de-France. Analyse technologique de l'industrie lithique du locus P., Mémoire de D.E.A., Université de Paris X.

THEVENIN A. (1997) - L“"Azilien" et les cultures à pointes à dos courbe : esquisse géographique et chronologique, Bulletin de la Soclété Préhistorique Française, t, 94, p. 393+411, 8 fig.

VALENTIN B. (1995) - Les groupes humains et leurs traditions an Tardiglaciaire dans le Bassin parisien. Apports de la technologie comparée, Thèse de Doctorat, Université de Paris I, 3 vol., ex. multigraph., $1106 \mathrm{p}$.

VALENTIN B. (1999) - Techniques et cultures : les chasseurs-cueitleurs du Tardiglaciaire dans le Sud et l'Ouest du Bassin parisien, in : A. Thévenin (éd.), L'Eurape des derniers chasseurs. Épipaléolithique et Mésolithique, Actes du 5* colloque international U.I.S.P.P., Commission XII, Grenoble, 18-23 sept. 1995, Paris, C.T.H.S., p. 201-212, 3 fig

VALENTIN B, JULIEN M. et BODU P. (1997) - La fin du Tardiglaciaire au sud-est de l'Ile-de-France : stratégie d'acquisition et de gestion des ressources lithiques, in: Le Paléolithique supérieur de l'Est de la France: de l'Aurignacien à l'Ahrensbourgien, Mémoire de la Société Archéologique Champenoise, 13, p. 105-117, $8 \mathrm{fig}$.

\section{Nicolas TEYSSANDIER \\ Doctorant, Université de Paris $X$} Laboratoire "Préhistoire et Technologie" Maison de l'Archéologie et de l'Ethnologie 92023 Nanterre cedex 